ARTICLE

DOI: $10.1038 / s 41467-017-01972-9$

\title{
Whole-exome sequencing identifies common and rare variant metabolic QTLs in a Middle Eastern population
}

Noha A. Yousri1,2, Khalid A. Fakhro1,3, Amal Robay', Juan L. Rodriguez-Flores (i) ${ }^{4}$, Robert P. Mohney (i) ${ }^{5}$, Hassina Zeriri ${ }^{1}$, Tala Odeh ${ }^{1}$, Sara Abdul Kader ${ }^{6}$, Eman K. Aldous ${ }^{7}$, Gaurav Thareja ${ }^{6}$, Manish Kumar ${ }^{6}$, Alya Al-Shakaki ${ }^{1}$, Omar M. Chidiac ${ }^{1}$, Yasmin A. Mohamoud ${ }^{7}$, Jason G. Mezey ${ }^{4}$, Joel A. Malek (D) 1,7, Ronald G. Crystal ${ }^{4} \&$ Karsten Suhre (ID ${ }^{6}$

Metabolomics-genome-wide association studies (mGWAS) have uncovered many metabolic quantitative trait loci (mQTLs) influencing human metabolic individuality, though predominantly in European cohorts. By combining whole-exome sequencing with a highresolution metabolomics profiling for a highly consanguineous Middle Eastern population, we discover 21 common variant and 12 functional rare variant mQTLs, of which $45 \%$ are novel altogether. We fine-map 10 common variant $\mathrm{mQTLs}$ to new metabolite ratio associations, and 11 common variant mQTLs to putative protein-altering variants. This is the first work to report common and rare variant $\mathrm{mQTLs}$ linked to diseases and/or pharmacological targets in a consanguineous Arab cohort, with wide implications for precision medicine in the Middle East.

\footnotetext{
${ }^{1}$ Genetic Medicine, Weill Cornell Medicine-Qatar, PO Box 24144 Doha, Qatar. ${ }^{2}$ Computer and Systems Engineering, Alexandria University, Alexandria, Egypt. ${ }^{3}$ Sidra Medical Research Center, Department of Human Genetics, PO Box 26999 Doha, Qatar. ${ }^{4}$ Genetic Medicine, Weill Cornell Medicine, New York, NY 10065, USA. ${ }^{5}$ Metabolon Inc, Durham, NC 27560, USA. ${ }^{6}$ Physiology and Biophysics, Weill Cornell Medicine-Qatar, PO Box 24144 Doha, Qatar. ${ }^{7}$ Genomics Core, Weill Cornell Medicine-Qatar, PO Box 24144 Doha, Qatar. Noha A. Yousri and Khalid A. Fakhro contributed equally to this work. Correspondence and requests for materials should be addressed to N.A.Y. (email: nay2005@qatar-med.cornell.edu) or to K.A.F. (email: kfakhro@sidra.org) or to K.S. (email: karsten@suhre.fr)
} 
M etabolites represent functional intermediates to the end phenotype, can be conserved over several years' time frame, and can uniquely identify individuals ${ }^{1}$. Several studies have shown that they are influenced by a combination of genetics and environment, the latter comprising both life style exposures and microbial interactions ${ }^{2}$. Recent technological improvements have enabled the accurate detection of thousands of metabolites (collectively, the metabolome), adding highly informative downstream read-outs supporting genetic and transcriptomic signatures in the study of personalized medicine ${ }^{2}$. With the abundance of such omics data, it will become possible to infer causal relationship between constitutive genetic variants and metabolite levels to accurately predict the likelihood of developing pathophysiologic signatures, as a normal individual progresses into a disease state.

To date, there have been several large-scale genome-wide association studies for metabolic traits (mGWAS) ${ }^{3-10}$ the largest (with broad non-targeted metabolomics) of which interrogated 7000 individuals of European ancestry and discovered 145 significant metabolomics quantitative trait loci $(\mathrm{mQTLs})^{11}$. While studies to date have uncovered hundreds of mQTLs, they have also faced certain limitations. First, they relied on imputed genotyping array data for the discovery of common variant mQTLs, where high-quality SNPs are in non-coding regions. More recently, next-generation sequencing (NGS) technologies have become more affordable and begun to identify protein-coding variants largely affecting metabolite levels, yet on a small scale of individuals or metabolites ${ }^{12-14}$ and very recently on a larger scale $^{15}$ (published while finalizing this manuscript). Second, metabolite detection platforms continue to rapidly improve, and deeper resolution can be gained today than previously possible. Third, as with lack of diversity in most GWAS studies ${ }^{16}$, most mGWAS to date have focused predominantly on populations of European descent ${ }^{7,8,11,17}$, and recently, Asian and African descent $^{18}$, yet little or no efforts have been described in Middle Eastern populations.

We present the first large-scale metabolomics exome-wide association study of a highly consanguineous Middle Eastern population, by combining 1,303 metabolites (from the most recent Metabolon DiscoveryHD4 platform) with deep wholeexome sequencing data of 614 Qataris for discovery, and imputed array data of another 382 Qataris for replication analysis. We integrate this data to discover loci affecting metabolites and metabolite ratios in this population, while fine-mapping loci to putative functional variants at or near sentinel SNPs (a sentinel SNP or sentinel metabolite refers to a lead SNP or a lead metabolite). Moreover, by leveraging elevated consanguinity in this population, we also discover rare variant loci associated with metabolite levels, underscoring the metabolic individuality of subjects from this ethnic population.

\section{Results}

Subject selection and genotyping. A total of 996 Qatari subjects were selected for this study (Table 1), of whom 614 were wholeexome sequenced (WES) for the discovery step, and 382 were array genotyped for replication. For the purpose of replication, both data sets were imputed using a reference set of 108 deeply covered, phased Qatari genomes. A total of 1,650,892 imputed exome variants were available for the analysis after Quality Control (see Methods). All samples were analyzed on Metabolon's DiscoveryHD4 platform, where a total of 1303 metabolites were detected (Supplementary Data 1). After applying strict QC, 826 metabolites (including 249 unknowns) remained for the association analysis (Fig. 1 gives a schematic representation of the study design).
Pre-discovery exploration of replication in Caucasians. As an investigation step prior to discovery, we attempted to replicate 145 previously known loci ${ }^{11}$ in the discovery cohort. We found exact or proxy SNPs (SNPs in LD) for 101 of 145 loci in our dataset, of which sentinel metabolites in 80 loci were matched to metabolites in our cohort. For 13 loci, we replicated associations between the reported SNP and the reported metabolite at a Bonferroni $p$-value $\left(p \leq 0.05 / 80=6.25 \times 10^{-4}\right)$, and for another 15 loci, we replicated the association at the same/proxy SNP but with another metabolite $\left(p<0.05 /(101 \times 826)=5.9 \times 10^{-7}\right)$ (Supplementary Data 2). In total, we replicated 28 loci (19.3\% of 145 loci, $35 \%$ of 80 replicable loci)—including 11 of the 20 most significant $\operatorname{loci}^{11}$.

21 common variant loci influence metabolites and ratios. To discover all metabolites associated with exome variants in 614 Qataris, 1.6 million imputed exome variants $(272,061$ SNPs after LD pruning) were tested for association with 826 metabolites (see Supplementary Fig. 1 for distribution of kinship-based heritability estimates for each super pathway). This step uncovered 3127 significant associations (Bonferroni $p \leq 2.2 \times 10^{-10}$ ) (Supplementary Data 7), with an average inflation factor of 0.98 (range: 0.83-1.05). Those associations collapsed into 19 independent loci (see Methods), which we attempted to replicate (based on the sentinel SNP and the sentinel metabolite) in the imputed array data set $(n=382)$ and found that 17 of them replicated $(p \leq 0.05 / 19=0.0026)$ and one nominally replicated at $p \leq 0.05$ (Table 2, Supplementary Datas 3-5).

In addition to single-metabolite-variant associations, we examined each locus to identify significantly associated metabolite ratios ${ }^{19}$. To limit multiple testing, two approaches were used to examine associations of metabolic ratios. First, we computed the associations between SNPs (within $100 \mathrm{~Kb}$ of the sentinel SNP from single-metabolite association analysis) and the ratio of the sentinel metabolite to all remaining 825 metabolites. Second, for all SNPs where two metabolites had been nominally associated in the discovery phase $\left(p \leq 10^{-4}\right)$ but in opposite directions (opposite beta signs), we computed the association of the given SNP to the ratio of that pair of metabolites. Both $p$-value and $p$ gain ${ }^{19}$ thresholds were used to find significant ratios. A total of 11 significant SNP to metabolite ratio associations were discovered with $p \leq 0.05 /(826 \times 18+826 \times 272,061)=2.2 \times 10^{-10}$ and $p$-gain $\geq\left(1 /(2 \times 0.05) \times(826 \times 18)=1.48 \times 10^{5}\right.$ (see Methods). Seven of these resulted in metabolic fine-mapping of seven of the loci discovered in the single-metabolite phase (i.e. where a ratio replaced the single metabolite as the sentinel association), whereas the remaining four were new associations at loci not associated with single metabolites. Only three of those four replicated in the cohort of 382 individuals (Table 2, and Supplementary Datas 3-5), resulting in a total of 10 SNP to metabolite ratio associations.

\section{Table 1 Demographics (sample characteristics)}

\begin{tabular}{ll} 
Demographic category & Attribute \\
\hline Gender (\% females) & $45 \%$ \\
Age (mean \pm s.d.) & $50.1 \pm 12.6$ \\
T2D (\% with diabetes) & $56 \%$ \\
BMI (mean \pm s.d.) & $32 \pm 6.6$ \\
Population information: & Q1:442 Q2:339 Q3:70, admixed: 54, \\
\#subjects & not assigned: 91 \\
Genotyping source: \#subjects & WES: 614, array genotyped: 382
\end{tabular}

WES whole-exome sequencing

Q1, Q2, and Q3 refer to Bedouin, Persian, and African ancestries, respectively, that present subpopulations of the people of Qatar ${ }^{36}$ 


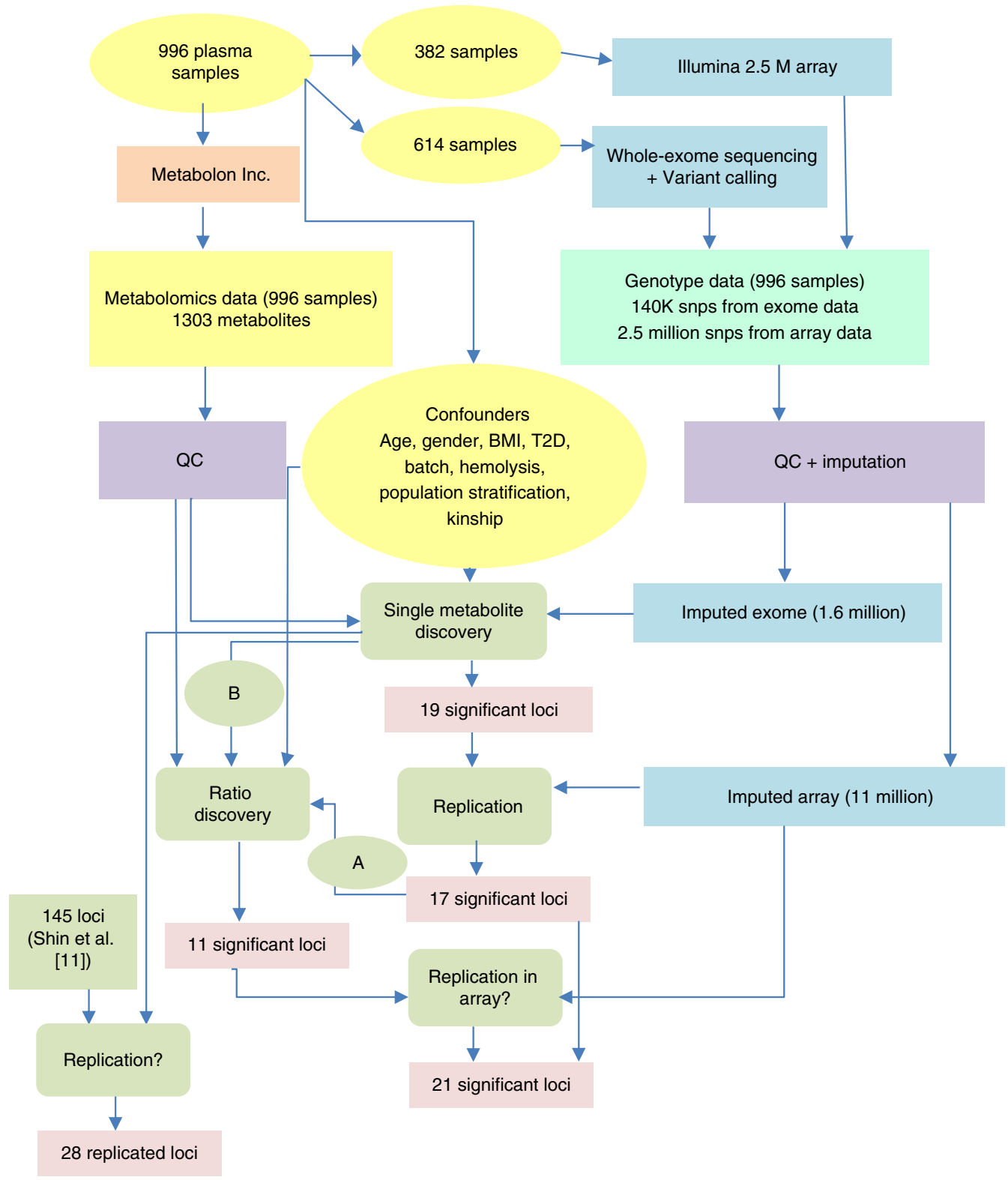

Fig. 1 Schematic view of the study design for the common variant analysis. ' $A$ ' indicates the first method for ratio computation, where we computed the associations between SNPs (within $100 \mathrm{~Kb}$ of the sentinel SNP from single-metabolite association analysis) and the ratio of the sentinel metabolite to all remaining 825 metabolites. 'B' indicates the second method for ratio computation, where for all SNPs for which two metabolites had been nominally associated in the discovery phase $\left(p \leq 10^{-4}\right)$, but in opposite directions (opposite beta signs), we computed the association of the given SNP to the ratio of that pair of metabolites

Combined, we discovered 21 unique metabolite and metabolite ratio quantitative trait loci (mQTLs) in Qataris (Fig. 2, and Supplementary Figs. 2 and 3). The variance explained by these genetic variants ranges from the highest value of $31 \%$ (5acetylamino-6-amino-3-methyluracil/1-methylxanthine with rs4646257 in NAT2 locus) to 7\% (association of undecanedioate with c15p90683852 in SEMA4B locus) with an average of $14.8 \%$ (Table 2, Fig. 3). Of the 21 loci, 7 (31\%) were unknown to studies that used older metabolomics platforms (studies prior to 2017) and are defined here as newly identified loci (four loci were concurrently identified in a study published while finalizing this manuscript ${ }^{15}$, and three loci are novel (Table 2, Supplementary Data 3). Five of those seven loci might be new due to the use of the new metabolomics platform, another two (TMPRSS11E and $S E M A 4 B)$ were not discovered in ref. ${ }^{11}$, and other known loci were fine-mapped to new metabolites, and not reported elsewhere (as rs2147896 in PYROXD2 with $N$-methylpipecolate, and UGT3A1 with X-24348). We explored the frequencies of the sentinel SNPs in the Genome Aggregation Database (gnomAD) ${ }^{20}$ (Supplementary Data 3), and found that among the seven loci, the sentinel SNP in two novel ones are not reported in the database (PHYHD1/NUP188, SEMA4B), and those in another two loci (SLC22A24, TTC38/PKDREJ) are rare in other populations.

All 10 reported metabolite ratio associations represent metabolic fine-mapping of loci discovered here or in previous studies. According to this cohort, those ratios associate more strongly than the single metabolites previously reported ${ }^{11,15}$. These include the association of rs4646257 in NAT2 locus with the ratio 5-acetylamino-6-amino-3-methyluracil/1-methylxanthine, rs375811360 in NAT8 locus with 2-aminooctanoate/X-12511 (X-12511 is possibly 2-acetamidooctanoic acid as identified by Metabolon), rs3756669 in UGT3A1 locus with X-24348/pregn- 
Table 221 Unique locus-metabolite pairs, indicating 7 newly identified and novel loci, 10 loci fine mapped with new metabolite ratio associations, and 11 protein coding variants

\begin{tabular}{|c|c|c|c|c|c|c|c|c|}
\hline Locus & rsID & Metabolite/Ratio & $p$-value (pgain ${ }^{a}$ ) & Beta & Annotation $^{b}$ & $\begin{array}{l}\mathbf{r} \\
(\%)^{c}\end{array}$ & $\begin{array}{l}\text { Ref/NL/ } \\
\text { NAS }^{\mathbf{d}}\end{array}$ & $\begin{array}{l}\text { Replication p- } \\
\text { value }^{\mathrm{e}}\end{array}$ \\
\hline NAT2 & rs4646257 & $\frac{\text { 5-acetylamino-6-amino-3-methyluracil }}{\text { 1-methylxanthine }}$ & $\begin{array}{l}2.23 \times 10^{-58} \\
\left(6.83 \times 10^{46}\right)\end{array}$ & 0.970 & $\begin{array}{l}\text { IG, NFS: } \\
\text { rs1801280, } \\
\text { p.lle114Thr }\end{array}$ & 31 & $(11,15)$ NAS & \\
\hline ACADS & $\begin{array}{l}\text { rs4921913 } \\
\text { rs1799958 }\end{array}$ & $\begin{array}{l}\text { 5-acetylamino-6-amino-3-methyluracil } \\
\text { Ethylmalonate }\end{array}$ & $\begin{array}{l}2.14 \times 10^{-12} \\
2.18 \times 10^{-53}\end{array}$ & $\begin{array}{l}0.499 \\
0.885\end{array}$ & p.Gly209Ser & $\begin{array}{l}7.8 \\
28.5\end{array}$ & $\begin{array}{l}(11,15) \\
(11,15)\end{array}$ & $\begin{array}{l}9.6 \times 10^{-9} \\
2.1 \times 10^{-21}\end{array}$ \\
\hline NAT8 & rs13538 & $\frac{\text { 2-aminooctanoate }}{\mathrm{X}-12511}$ & $\begin{array}{l}4.4 \times 10^{-47} \\
\left(8.6 \times 10^{35}\right)\end{array}$ & -0.781 & p.Phe143Ser & 26.6 & $(11,15)$ NAS & \\
\hline $\begin{array}{l}\text { TMPRSS11E } \\
\text { SLCO1B1 } \\
\text { PYROXD2 }\end{array}$ & $\begin{array}{l}\text { rs13538 } \\
\text { rs34109652 } \\
\text { rs4149056 } \\
\text { rs2147896 }\end{array}$ & $\begin{array}{l}\text { N-acetylcitrulline } \\
\text { X-11491 (deoxycholic acid glucuronide or isomer) } \\
\text { glycochenodeoxycholate glucuronide (1) } \\
\text { N-methylpipecolate }\end{array}$ & $\begin{array}{l}5.5 \times 10^{-32} \\
3.28 \times 10^{-35} \\
3.06 \times 10^{-31} \\
9.13 \times 10^{-26}\end{array}$ & $\begin{array}{l}0.780 \\
-0.737 \\
0.833 \\
-0.663\end{array}$ & $\begin{array}{l}\text { INT } \\
\text { p.Val174Ala } \\
\text { p.Met461Thr }\end{array}$ & $\begin{array}{l}19.6 \\
21.4 \\
18.5 \\
18.3\end{array}$ & $\begin{array}{l}(11,15) \\
(15) \\
\left(\begin{array}{ll}11,15 \\
)\end{array}\right) \text { NAS } \\
\left({ }^{11}\right) \text { NAS }\end{array}$ & $\begin{array}{l}3.7 \times 10^{-21} \\
3.8 \times 10^{-25} \\
5.5 \times 10^{-22} \\
7.4 \times 10^{-19}\end{array}$ \\
\hline \multirow[t]{2}{*}{ UGT3A1 } & rs3756669 & $\frac{\mathrm{X}-24348}{\text { pregn steroid monosulfate }^{*}}$ & $\begin{array}{l}1.55 \times 10^{-25} \\
\left(4.02 \times 10^{12}\right)\end{array}$ & -0.915 & p.Cys121Gly & 16.6 & $\left({ }^{11}\right)$ NAS & \\
\hline & rs3756669 & $x-24348$ & $6.25 \times 10^{-13}$ & -0.712 & & 8.5 & $\left({ }^{11}\right)$ NAS & $1.01 \times 10^{-9}$ \\
\hline \multirow[t]{2}{*}{ FADS2 } & rs28456 & $\frac{1 \text {-(1-enyl-palmitoyl)-2-arachi-donoyl-GPC }(\mathrm{P}-16: 0 / 20: 4)^{*}}{\mathrm{X}-24438(\mathrm{PC}(\mathrm{P}-16: 0 / 20: 3))}$ & $\begin{array}{l}9.81 \times 10^{-25} \\
\left(5.38 \times 10^{16}\right)\end{array}$ & -0.641 & INT & 17.8 & $(11,15)$ NAS & \\
\hline & rs174560 & $X-24439\left(P E(P-16: 0 / 20: 3)^{*}\right)$ & $9.09 \times 10^{-14}$ & 0.457 & INT & 8.9 & $(11,15)$ & $1.6 \times 10^{-3}$ \\
\hline$A G \times T 2$ & rs37370 & 3-aminoisobutyrate & $8.45 \times 10^{-21}$ & 0.810 & p.Ser102Asn & 13 & $\left({ }^{15}\right)$ & $4.3 \times 10^{-10}$ \\
\hline PHYHDI $^{\dagger}$ & rs181856093 & X-22145 (2'-O-methyluridine) & $3.29 \times 10^{-20}$ & 0.531 & $\begin{array}{l}\text { INT, NFS: } \\
\text { rs2302811, } \\
\text { INS: } \\
\text { c.3662-4A > } \\
\text { G }\end{array}$ & 12.7 & $\mathrm{NL}$ & $1.3 \times 10^{-11}$ \\
\hline THEM4 & rs6690449 & $\frac{X-18921}{X-23680}$ & $\begin{array}{l}9.35 \times 10^{-20} \\
\left(2.29 \times 10^{9}\right)\end{array}$ & -0.547 & INT & 14.1 & $(11,15)$ NAS & \\
\hline UGT1A1 & $\begin{array}{l}\text { rs2999534 } \\
\text { rs78461713 }\end{array}$ & $\begin{array}{l}\text { X-23293 } \\
\text { bilirubin }(E, E)^{*}\end{array}$ & $\begin{array}{l}1.15 \times 10^{-11} \\
5.1 \times 10^{-17}\end{array}$ & $\begin{array}{l}0.426 \\
0.484\end{array}$ & $\begin{array}{l}\text { IG } \\
\text { INT }\end{array}$ & $\begin{array}{l}7.2 \\
10.6\end{array}$ & $\begin{array}{l}(11,15) \\
(11,15)\end{array}$ & $\begin{array}{l}2.05 \times 10^{-4} \\
1.4 \times 10^{-12}\end{array}$ \\
\hline SULT2A1 & rs62129970 & $\frac{\mathrm{X}-11440(\text { tentatively steroid) }}{\text { 4-androsten-3alpha, 17alpha-diol monosulfate(2) }} 62$ & $\begin{array}{l}7.59 \times 10^{-17} \\
\left(7.33 \times 10^{10}\right)\end{array}$ & -0.951 & IG & 11 & $\left({ }^{11}\right)$ & $1.4 \times 10^{-17}$ \\
\hline \multirow[t]{2}{*}{$S L C 22 A 24^{f}$} & rs78176967 & $\frac{\text { X-22379(androsterone glucuronide) }}{21-\text { hydroxypregnenolone disulfate }}$ & $\begin{array}{l}1.43 \times 10^{-16} \\
\left(6.75 \times 10^{6}\right)\end{array}$ & 0.995 & IG & 10.8 & $\left({ }^{15}\right)$, NAS & \\
\hline & rs61285056 & X-22379 (androsterone glucuronide) & $9.11 \times 10^{-11}$ & 0.765 & INT & 6.9 & $\left({ }^{15}\right)$ & $5.2 \times 10^{-8}$ \\
\hline SPTLC1P4 ${ }^{f}$ & rs2069258 & $\frac{X-23293}{\text { cis-4-decenoyl carnitine }}$ & $\begin{array}{l}1.71 \times 10^{-16} \\
\left(3.69 \times 10^{8}\right)\end{array}$ & 0.425 & IG & 10.6 & $\mathrm{NL}$ & $2.4 \times 10^{-6}$ \\
\hline TTC38 ${ }^{f}$ & rs117135869 & $\frac{X-22162}{X-24513}$ & $\begin{array}{l}4.264 \times 10^{-16} \\
\left(2.06 \times 10^{5}\right)\end{array}$ & 0.623 & p.Ala12Val & 10.4 & $\left({ }^{15}\right)$ NAS & \\
\hline $\begin{array}{l}S L C 22 A 5 \\
C C B L 2^{f, g}\end{array}$ & $\begin{array}{l}\text { rs117135869 } \\
\text { rs274554 } \\
\text { rs7530513 }\end{array}$ & $\begin{array}{l}\text { X-22162 } \\
\text { Tryptophan betaine } \\
\text { Imidazole lactate }\end{array}$ & $\begin{array}{l}8.8 \times 10^{-11} \\
1.05 \times 10^{-13} \\
5.6 \times 10^{-13}\end{array}$ & $\begin{array}{l}0.616 \\
-0.430 \\
0.425\end{array}$ & $\begin{array}{l}\text { INT } \\
\text { INT }\end{array}$ & $\begin{array}{l}6.7 \\
8.6 \\
8.1\end{array}$ & $\begin{array}{l}\left(\begin{array}{l}15 \\
) \\
(11,15\end{array}\right) \\
(15)\end{array}$ & $\begin{array}{l}1.7 \times 10^{-4} \\
3.3 \times 10^{-10} \\
3.2 \times 10^{-2}\end{array}$ \\
\hline SLC17A1 & rs1165196 & $\frac{X-12824(\text { hexanoylglutamine })}{X-16087}$ & $\begin{array}{l}1.46 \times 10^{-12} \\
\left(1.30 \times 10^{6}\right)\end{array}$ & -0.543 & p.Thr269lle & 8.8 & $(11,15)$ NAS & $8.5 \times 10^{-8}$ \\
\hline CYP3A5 & rs776746 & $X-12063$ & $1.54 \times 10^{-12}$ & -0.620 & $\begin{array}{l}\text { SA, c.219- } \\
237 A>G\end{array}$ & 7.8 & $(11,15)$ & $1.2 \times 10^{-12}$ \\
\hline SEMA4B ${ }^{f}$ & c15p90683852 & Undecanedioate & $1.18 \times 10^{-10}$ & 0.421 & IG & 7 & $\mathrm{NL}$ & $9.7 \times 10^{-4}$ \\
\hline \multicolumn{9}{|c|}{ 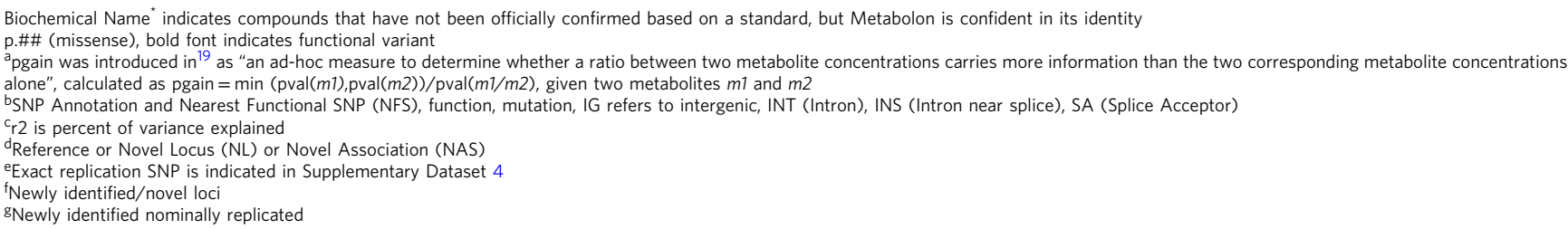 } \\
\hline
\end{tabular}

steroid-monosulfate, rs28456 in FADS2 locus with 1-(1-enylpalmitoyl)-2-arachidonoyl-GPC(P-16:0/20:4)/X-24438 (X-24428 is $\mathrm{PC}(\mathrm{P}-16: 0 / 20: 3)$ as identified by Metabolon), rs6690449 in THEM4 locus with X-18921/X-23680 and rs1165196 in SLC17A1 locus with X-12824/X-16087 (X-12824 is hexanoylglutamine as identified by Metabolon) (see expanded loci associations-Supplementary Data 5). Notably, the variance explained by ratios was much higher than that explained by any single metabolite for the same locus (Table 2); for example, for the NAT2 locus, the variance explained by the ratio of metabolites is 3.9-fold greater than that explained by the single metabolite; similarly, 1.9-fold greater in the FADS2, UGT3A1 and THEM4 loci, 1.5-fold greater in SLC22A24 and TTC38/PKDREJ loci, and 1.3-fold greater in the NAT8 locus (comparison of ratios to single metabolites-Fig. 4).

We investigated the 21 loci for known eQTLs and disease or pharmacological associations using several databases (biological details are in Supplementary Data 6): GTEx portal (version 2.1, Build \#201), OMIM database ${ }^{21}$, Orphanet disease database, CHEMBL targets database, PharmaGKB ${ }^{22}$ and SNIPA (see URLs in Methods). We found that seven sentinel SNPs are in genes encoding enzymes or transporters. Additionally, 16 sentinel SNPs are known eQTLs, including 5 at novel and newly identified loci (PHYHD1/NUP188, TTC38/PKDREJ, TMPRSS11E, SPTLC1P4/ $A L 591893.1, C C B L 2)$. Moreover, 10 loci contain genes linked to diseases, including 4 of the newly identified loci; namely, NUP188 


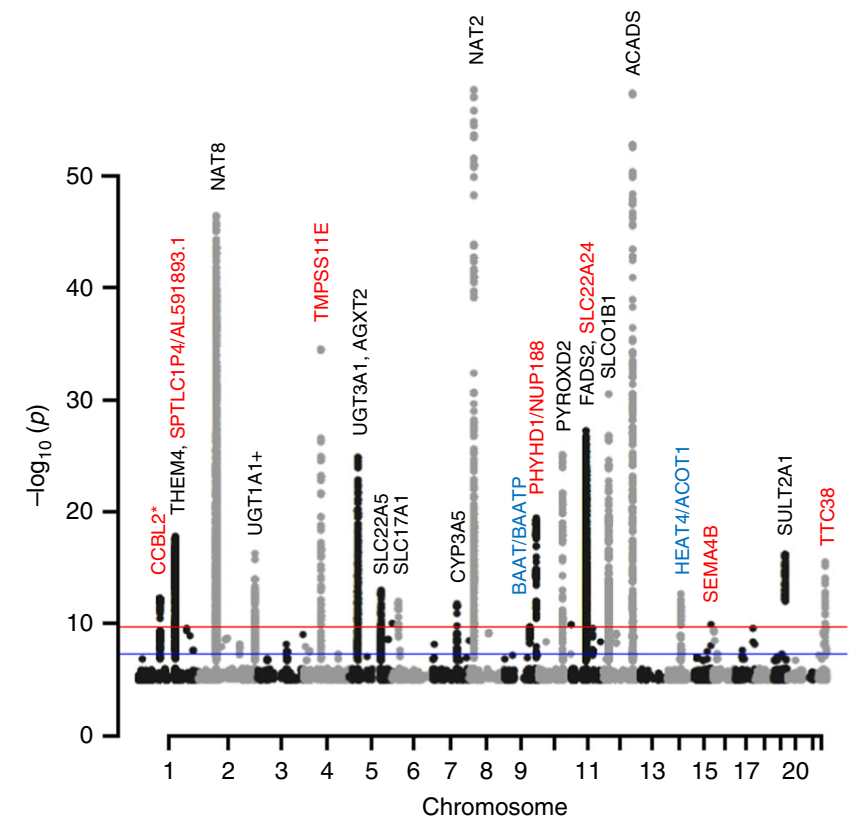

Fig. 2 Manhattan plot for the discovered loci. The red line indicates the Bonferroni threshold $\left(2.2 \times 10^{-10}\right)$ and the blue line indicates the genome wide significance threshold $\left(5 \times 10^{-8}\right)$. The newly identified/novel replicated loci are typed in red, and the non-replicated loci are in blue. Unnamed loci at the borderline $\left(2.2 \times 10^{-10}\right)$ are associations with ratios with a $p$-gain below the threshold. *stands for nominally replicated loci

has a role in heterotaxy ${ }^{23}, S E M A 4 B$ in hypoxia and lung cancer ${ }^{24}$, CCBL2 in neurodegenerative diseases, and TMPRSS11E in squamous cell carcinomas ${ }^{25}$. Finally, genes in 4 loci have been previously studied for effect on drugs.

Fine-mapping identifies functional variant associations. The availability of WES data in this study population allowed us to investigate if specific loci could be explained by functional (protein-altering) variants. Among the 21 loci, 11 (51\%) harbored SNPs that were protein-altering or splice variants (Table 2, Supplementary Data 3). Two of these are among the novel or newly identified loci: the splice variant c.3662-4 A $>\mathrm{G}$ (rs2302811) in NUP188 (PHYHD1/NUP188 locus), association with X-22145 (2'-O-methyluridine) and the missense variant $\mathrm{p}$. Ala12Val (rs117135869) in TTC38 (TTC38/PKDREJ locus) association with X-22162/X-24513, and the remaining nine are in previously reported mQTLs (Table 2). More importantly is the missense p.Cys121Gly (rs3756669) in UGT3A1 (Fig. 5a) which was found to be associated with undetected levels of X-24348 (these appear as missing values in the raw unprocessed metabolite levels) in the subjects which are homozygotes for the mutation, thus a potential loss of function in a pathway possibly related to the metabolite production ${ }^{26}$ (see Discussion). Additionally, the association of the missense variant p.Gly209Ser (rs1799958) in ACADS (Fig. 5b) to ethylmalonate levels (concurrently reported $^{15}$ ) is of clinical implication due to the association of the mutated allele with mild SCAD deficiency (MIM: 606885.0007).

Rare variants associated with metabolites in Qataris. In addition to common-variant mQTL discovery, we hypothesized that with a modest sample size, one may find rare functional variants influencing metabolite levels in homozygous state shared by more than one individual due to high levels of consanguinity. We selected high-quality protein-altering rare variants $(\mathrm{MAF}<5 \% ; n$

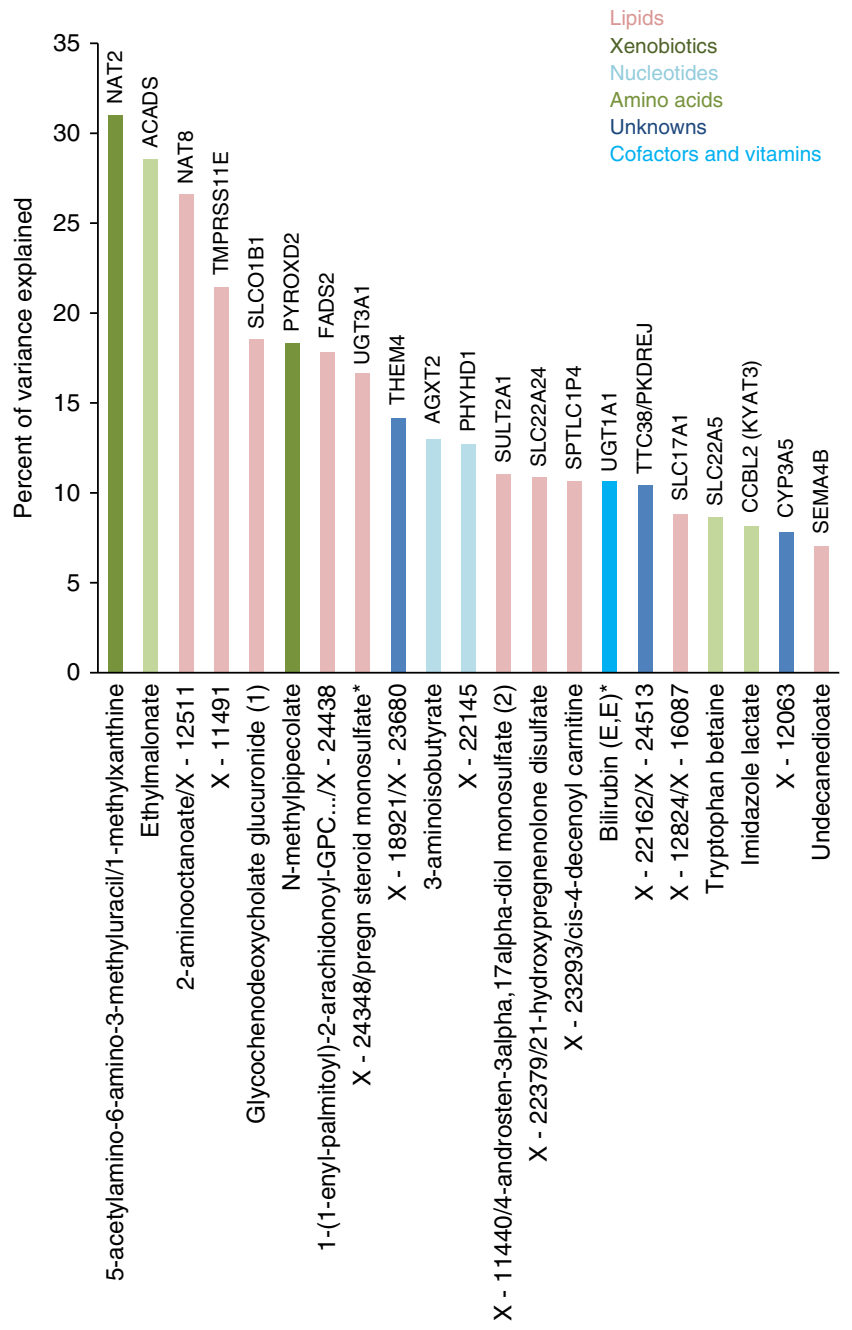

Fig. 3 Percent of variance explained in the 21 loci. The height of a column bar indicates the percent of variance explained for each locus, loci genes are indicated above the column bar, and the metabolite/ratio on the $X$-axis. Bars are colored according to Metabolon pathway specified for the metabolites associated with the locus. Biochemical Name* indicates compounds that have not been officially confirmed based on a standard, but Metabolon is confident in its identity

$=21,933$ SNPs, see Methods). First, we performed gene-based burden testing for all genes harboring at least one of these rare variants $\left(n=9823\right.$ genes; Bonferroni $\left.p \leq 6.16 \times 10^{-9}\right)$; and second, we tested single variant associations for SNPs with at least two homozygotes for the rare variant $(n=2660$ SNPs in 2119 genes; Bonferroni $p \leq 2.27 \times 10^{-8}$ ). In this analysis, we focused on rare, homozygous variants shared by two or more individuals, whose metabolite values were at either tail of the distribution (highest or lowest). After stringent QC and filtering, we discovered two genes with rare variants influencing metabolite levels through geneburden analysis. In contrast to burden testing, we identified 10 variants significantly influencing metabolite levels through singlevariant testing (Table 3, Fig. 6, Supplementary Fig. 5).

\section{Discussion}

We describe the first large-scale ( $n=996$ individuals) metabolite association study in a Middle Eastern population, combining deep WES data with an updated metabolomics platform. Altogether, we discover and replicate 21 common variant mQTLs. Amongst those, seven are novel or newly identified loci (associations that 
a

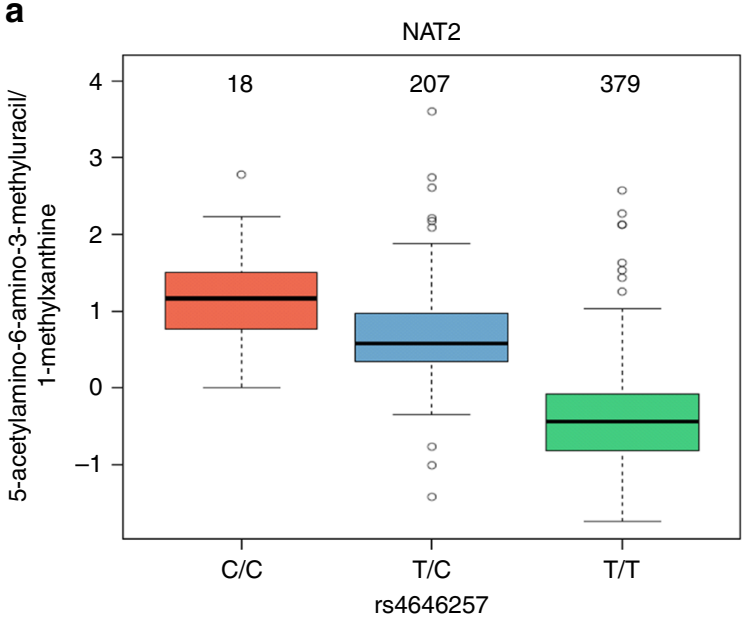

C

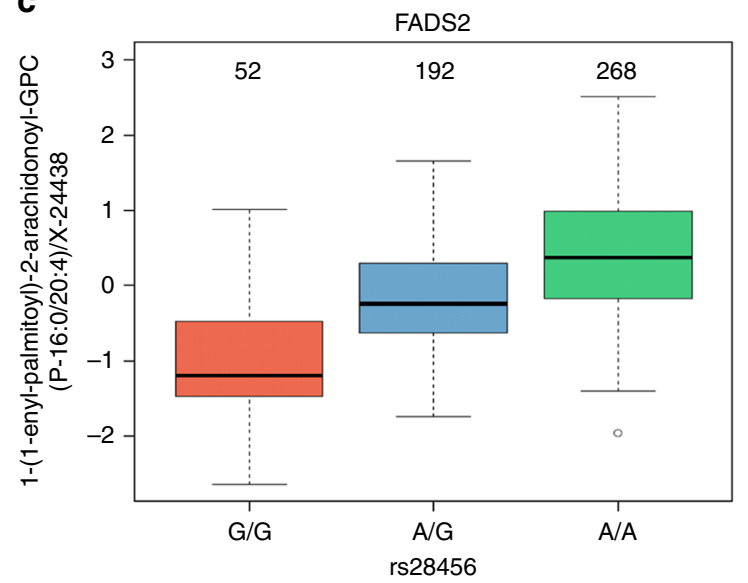

e

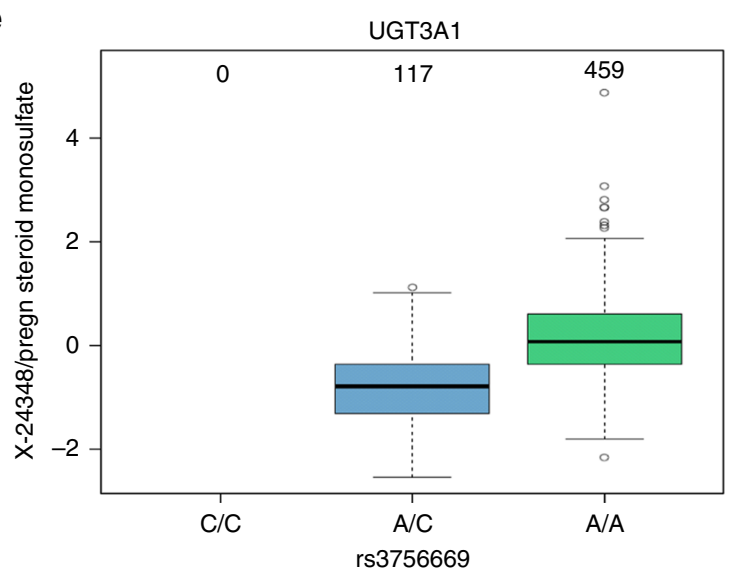

b

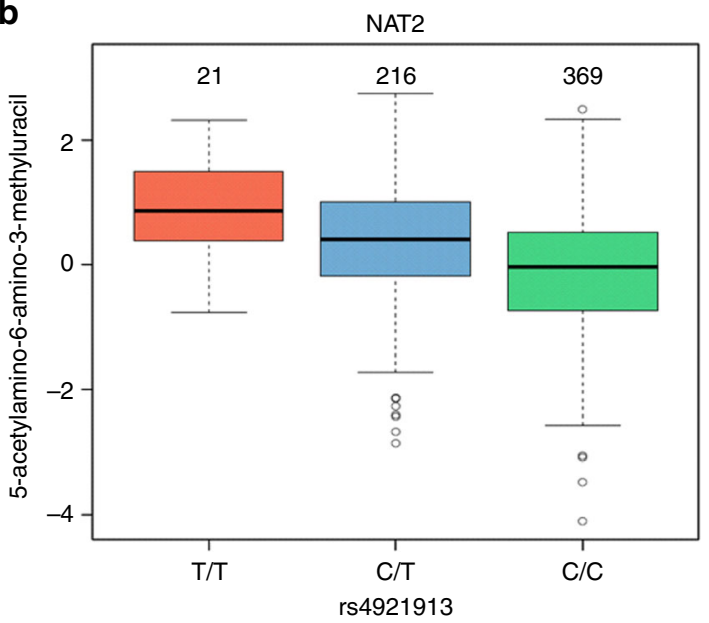

d

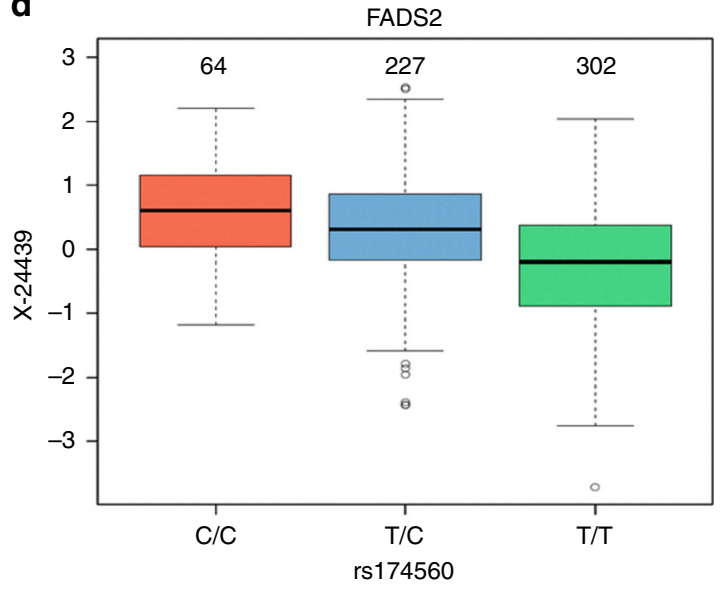

f

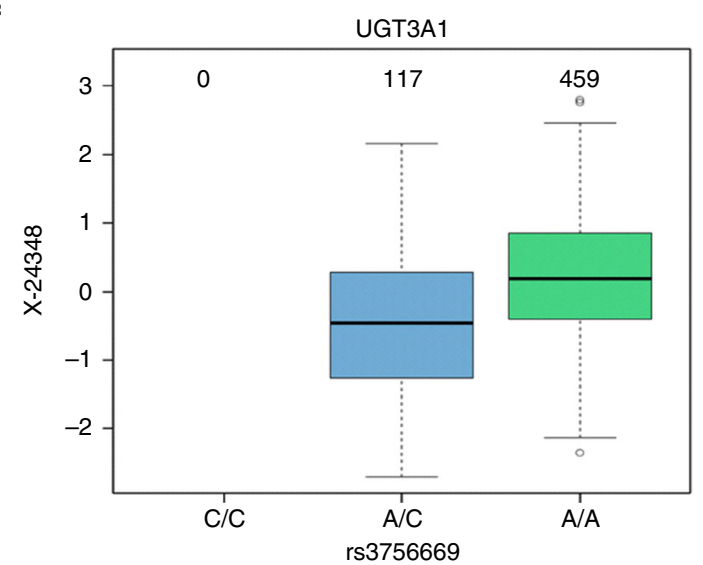

Fig. 4 Boxplots for the loci NAT2, FADS2, and UGT3A1. Boxplots showing metabolite/ratio levels and number of samples for each genotype group and comparing ratios to single metabolites for NAT2 $(\mathbf{a}, \mathbf{b})$, FADS2 $(\mathbf{c}, \mathbf{d})$, and UGT3A1 $(\mathbf{e}, \mathbf{f})$ loci, where the percent of variance explained by the ratio is 3.9-, 1.99-, and 1.94-fold greater, respectively, than that explained by the single metabolite

have been concurrently discovered ${ }^{15}$ and completely new ones, as indicated in Table 2), and 10 represent fine-mapping of previously reported loci to new metabolite ratios that associate more significantly than previously discovered single metabolites. Importantly, by having deep exome data, we fine-map a total of 11 loci to candidate protein-altering variants, with biological implications described below. Further, in the rare variant spectrum, we present 12 novel mQTL associations, all not previously reported.
Being able to replicate $35 \%$ of the 80 replicable loci reported in the largest mGWAS study to date ${ }^{11}$ with one tenth the sample size may be attributable to the high levels of consanguinity in our population, which allows for sufficient numbers of alternate allele homozygotes to be present for metabolic associations. Loci replicated among different ethnicities are likely to belong to pathways common to human metabolism (7 out of the 10 most significant loci reported in $^{11}-P Y R O X D 2$, ACADS, NAT8, 

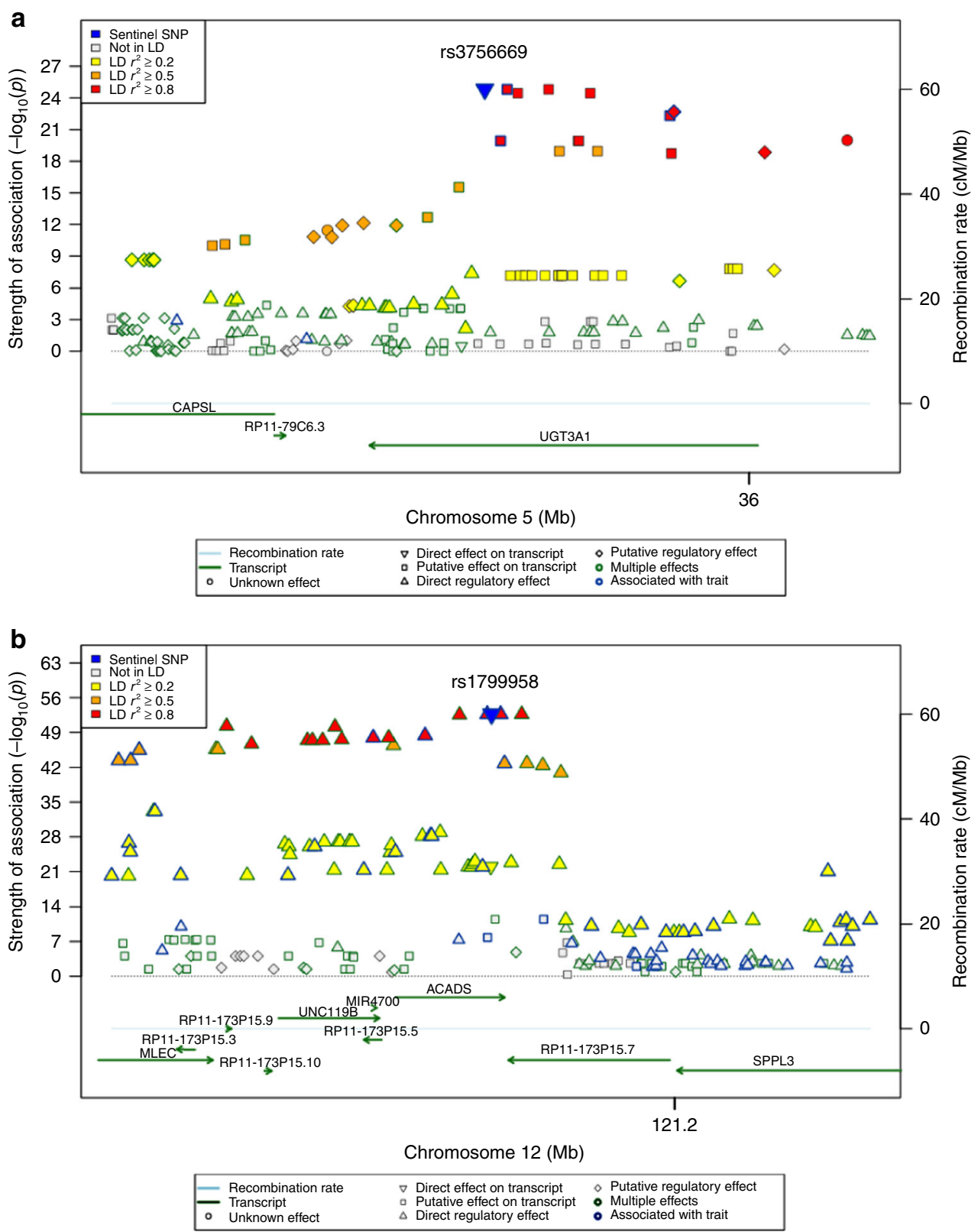

Fig. 5 Regional association plots for the loci UGT3A1 and ACADS. UGT3A1 (a) and ACADS (b) loci missense SNPs showing the strength of the association $(-\log 10$ ( $p$-value $))$ for $X-24348 /$ pregn-steroid-monosulfate* and ethylmalonate, respectively, on the $Y$-axis and the genes on the $X$-axis. The colors correspond to different LD thresholds, where LD is computed between the sentinel SNP (lowest $p$-value, colored in blue) and all SNPs. Shapes of markers correspond to their functionality as described in the legend

FADS2, SLCO1B1, SULT2A1, and UGT1A1-being among the top associations in our study).

Several of the newly discovered associations may reveal putative biological links between SNPs and metabolites. One such example is the association of rs34109652 in TMPRSS11E with X-11491 (tentatively identified by Metabolon as the bile acid deoxycholic acid glucuronide). This particular SNP had been reported as an eQTL for UGT2B15 (GTEx version 4.1, Build \#201), a gene at which $\mathrm{CpG}$ methylation was also significantly associated with X11491 levels $^{27}$, consistent with the gene's putative function of conjugating bile acids (GenAtlas(http://genatlas.medecine.univparis5.fr/fiche.php? $\mathrm{n}=6643)$ ). Another biological association is that of the missense variant rs117135869 (p.Ala12Val) in TTC38 (TTC38/PKDREJ locus) associating significantly to X-22162, and more significantly, to the ratio of this metabolite to X-24513. TTC38 (Tetratricopeptide Repeat Domain 38) expression is significantly positively correlated with age $\mathrm{e}^{28}$, and levels of both metabolites are also significantly associated with age by regression analysis in our cohort (see Methods). Additionally, X-24513 is significantly partially correlated with C-mannosyltryptophan (also known as C-glycosyltryptophan) (see Methods), which was reported to be associated with aging ${ }^{29}$. More interestingly, the fact that this locus encompasses PKDREJ (Polycystic Kidney Disease and Receptor for Egg Jelly related protein) gene (regional association plot-Supplementary Fig. 3), a homolog of PKD genes associating with kidney disease ${ }^{30}$, and that X-24513 might be similar in characteristics to C-mannosyltryptophan, reportedly elevated in chronic kidney disease ${ }^{31}$, highlights the importance of investigating the biological implication of this association in relation to both kidney diseases and ageing. Finally, the association of rs7530513 in the aminotransferase CCBL2 (Cysteine-S-conjugate beta-lyase 2, also known as KYAT3 
(Kynurenine-oxoglutarate transaminase 3) with imidazole lactate is another biologically relevant association; CCBL2 enzyme [EC2.6.1.7] has a transaminase activity towards histidine (Uniprot ${ }^{32}$ ) and it has been reported that histidine transaminase [EC2.6.1.38], takes L-histidine and 2-oxyglutarate as substrates and produces imidazole pyruvate that is later converted to imidazole lactate ${ }^{33}$. However, we are unable to confirm that the same histidine transaminase is the one involved in both processes.

A main advantage of using WES data in an association analysis is the enhanced resolution at associated loci that could reveal protein-altering variants affecting metabolite levels. We were able to identify such sentinel SNPs for over half of all loci (11 of 21), possibly revealing functional relationships between genes harboring these variants and their associated metabolites. One such example is the fine-mapping of a previously identified signal in the $3^{\prime} \mathrm{UTR}$ of ACADS to a missense mutation (rs1799958, p.Gly209Ser) in exon 6 (Fig. 5b). This mutation (MIM 606885.0007) has been reported independently as causing mild SCAD deficiency, with the mutant allele predicted to have $85 \%$ of wild-type activity ${ }^{34}$. Further, the populationfrequency of this variant (MAF $25 \%$ in GnomAD, 37.8\% in Qataris) is consistent with it having been the mild functional mutation in previously reported association signals between this locus and levels of both ethylmalonate and butyrylcarnitine, metabolites whose levels are known to be perturbed in SCAD deficiency ${ }^{35}$.

Another important finding is at the UGT3A1 locus, where, in contrast to previous studies that reported an association of the intergenic SNP rs10491431 with steroid levels, we uncovered an association of the missense mutation rs3756669 (p.Cys121Gly) in UGT3A1 with the unknown metabolite X-24348 and more strongly with the ratio of X-24348 to pregn-steroid-monosulfate (Figs. 4 and 5). This unknown has shown significant partial correlation to two other metabolites that also significantly correlate with steroids (Supplementary Fig. 4a, see Methods). The missense mutation therefore possibly interferes with native gene function related to the production of this metabolite, since all minor homozygotes for this SNP had missing values of X-24348
(Supplementary Fig. 2) $\quad\left(p\right.$-value $=5.94 \times 10^{-12}$ for random occurrence of missing values, see Methods), suggesting the amino-acid substitution leads to loss of function of the gene. This hypothesis is supported by previous functional evidence demonstrating that this mutation leads to diminished UGT3A1 glucuronidation activity ${ }^{26}$, especially of bile acids and estradiol. Metabolon has also recently studied this unknown and found that it might be a form of $\mathrm{N}$-acetylglucosamine modification of a pregnandiol, yet cannot be confirmed at the time being. Together, the data suggest the unknown metabolite may itself be a conjugated steroid, which requires this conjugation to appear at detectable levels in the blood. Notably, the mutation appears to have population-specific allele distribution, having been observed in up to $40 \%$ of Asians, $15 \%$ of Europeans, but only $3 \%$ of Africans ${ }^{26}$ - a distribution which is maintained in our study cohort (overall Qatari MAF 12.8\%), being present in Q1 and Q2 Qataris (Bedouin and Persian/South Asian), but not in Q3, who are of African descent ${ }^{36}$.

In addition to identifying functional SNPs, the use of the updated metabolomics platform (DiscoveryHD4) enabled the refinement of previously reported loci to new metabolites or metabolite ratios in the same or in different biological pathway from those originally reported ${ }^{11}$. For example, NAT2 encodes an arylamine transferase that controls the conversion of paraxanthine to 5-acetylamino-6-amino-3-methyluracil ${ }^{33}$, and its locus has previously been reported to be associated with 1methylxanthine. In this study, we discovered a stronger association with the ratios 5-acetylamino-6-amino-3-methyluracil/1methylxanthine and 5-acetylamino-6-amino-3-methyluracil/ paraxanthine, which are consistent with the known activity of the NAT2 enzyme in caffeine metabolism (see network of metabolites linked to NAT2, and KEGG pathway in Supplementary Fig. 4b, c). Being the top most significant association in this population in comparison to the top most ones previously reported ${ }^{11,15}$, it might have several biological implications for the studied population. It is thus worthy to note that the two missense SNPs at this locus are identified in OMIM as associated with the rate of acetylation, presenting a possible mechanism for affecting drug

Table 312 novel functional rare variant mQTLs

Gene-based burden test

\begin{tabular}{|c|c|c|c|c|c|c|}
\hline $\begin{array}{l}\text { SNP name } \\
\text { (c\#chr p\#position) }\end{array}$ & rsID & Gene & Metabolite & EA/OA & C-MAF & $p$-value \\
\hline $\begin{array}{l}c 4 p 57221348 \\
\text { c4p57248716 } \\
\text { c4p57250285 } \\
\text { c12p56075599 } \\
\text { c12p56075915 } \\
\text { c12p56077768 }\end{array}$ & $\begin{array}{l}\text { rs3796543 } \\
\text { rs34228795 } \\
\text { rs34543011 } \\
\text { rs199581976 } \\
\text { rs75289684 } \\
\text { rs115687886 }\end{array}$ & $\begin{array}{l}A A S D H \\
\text { AASDH } \\
\text { AASDH } \\
\text { METTL7B } \\
\text { METTL7B } \\
\text { METTL7B }\end{array}$ & $\begin{array}{l}\text { Thyroxine } \\
\text { Thyroxine } \\
\text { Thyroxine } \\
\text { Androsterone sulfate } \\
\text { Androsterone sulfate } \\
\text { Androsterone sulfate }\end{array}$ & $\begin{array}{l}\mathrm{C} / \mathrm{T} \\
\mathrm{C} / \mathrm{G} \\
\mathrm{C} / \mathrm{T} \\
\mathrm{T} / \mathrm{C} \\
\mathrm{A} / \mathrm{C} \\
\mathrm{T} / \mathrm{C}\end{array}$ & 0.036 & $4.78 \times 10^{-09}$ \\
\hline
\end{tabular}

Single-variant analysis

\begin{tabular}{|c|c|c|c|c|c|c|c|c|}
\hline $\begin{array}{l}\text { SNP name } \\
\text { (c\#chr p\#position) }\end{array}$ & rsID & Gene & Metabolite & EA/OA & EAF & $\mathbf{N}$ & Beta (s.e) & p-value \\
\hline c12p11506114* & - & PRB1 & Mannose & $\mathrm{T} / \mathrm{G}$ & 0.013 & 576 & $-1.32(0.192)$ & $5.99 \times 10^{-12}$ \\
\hline c15p89398112 & rs150988100 & $A C A N$ & X-12844 (glucuronidated steroid) & $A / G$ & 0.006 & 581 & $-2.05(0.323)$ & $1.88 \times 10^{-10}$ \\
\hline c2p26760624 & rs56332208 & OTOF & Retinol (Vitamin A) & $\mathrm{T} / \mathrm{C}$ & 0.0153 & 586 & $-1.18(0.19)$ & $4.83 \times 10^{-10}$ \\
\hline c15p89398112 & rs150988100 & ACAN & $X-09789$ & $A / G$ & 0.0059 & 592 & $-2.25(0.373)$ & $1.56 \times 10^{-09}$ \\
\hline c18p48256030 & - & MAPK4 & X-21365 (N-trimethyl 5-aminovalerate) & $\mathrm{C} / \mathrm{G}$ & 0.0161 & 589 & $-1.27(0.22)$ & $6.84 \times 10^{-09}$ \\
\hline c20p18295959 & rs377301648 & ZNF133 & 3-methyl-2-oxovalerate & $\mathrm{C} / \mathrm{T}$ & 0.0160 & 592 & $-1.15(0.2)$ & $9.36 \times 10^{-09}$ \\
\hline c11p18158958 & rs61733595 & MRGPRX3 & Tryptophan & $\mathrm{T} / \mathrm{C}$ & 0.0084 & 592 & $-1.43(0.25)$ & $1.01 \times 10^{-08}$ \\
\hline c12p55688833 & rs372117452 & OR6C6 & Androsterone sulfate & $G / A$ & 0.012 & 590 & $-1.37(0.24)$ & $1.09 \times 10^{-08}$ \\
\hline c12p56086993 & rs144983062 & ITGA7 & Androsterone sulfate & $\mathrm{C} / \mathrm{T}$ & 0.023 & 590 & $-1.02(0.18)$ & $1.25 \times 10^{-08}$ \\
\hline c18p48256030 & - & MAPK4 & Tryptophan & $\mathrm{G} / \mathrm{C}$ & 0.016 & 592 & $-1.11(0.19)$ & $1.32 \times 10^{-08}$ \\
\hline
\end{tabular}

EA effective allele, $O A$ observed allele, EAF effective allele frequency, CMAF cumulative minor allele frequency

All SNPs have a call rate of $100 \%$ except for the SNP marked $\left({ }^{\star}\right)$, which has a call rate of $97 \%$ 
a

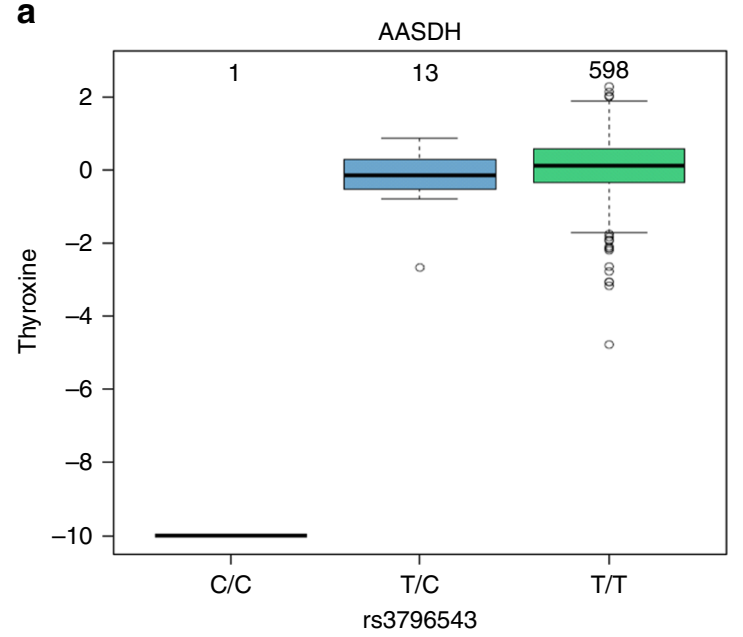

C

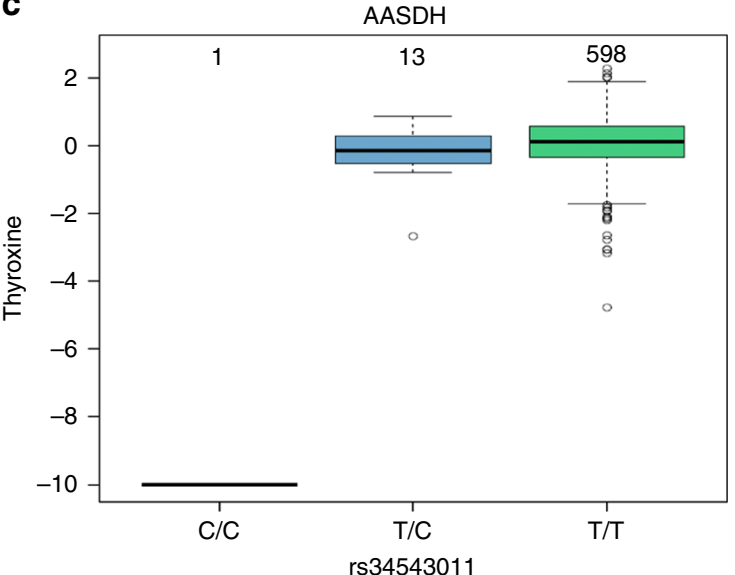

e

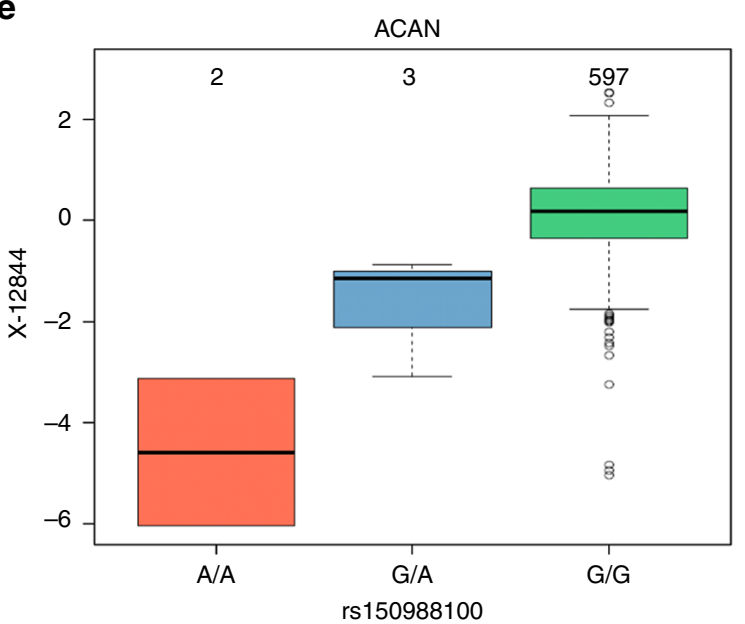

b

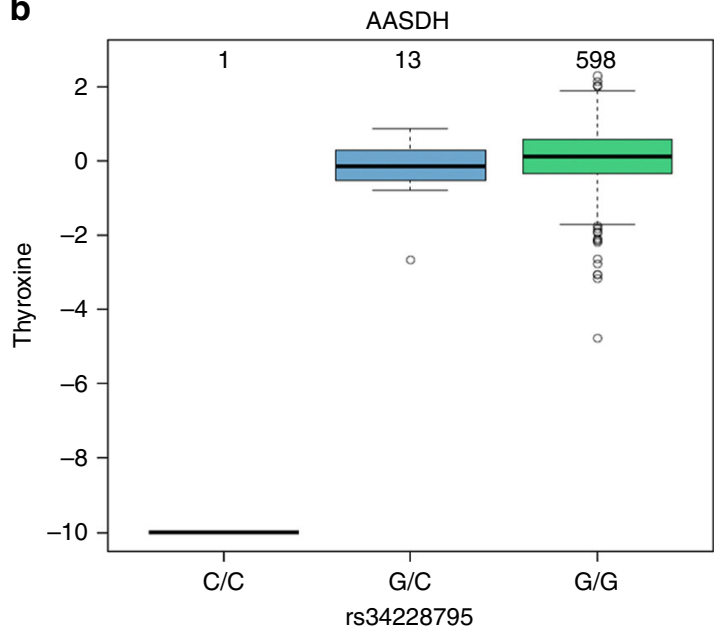

d

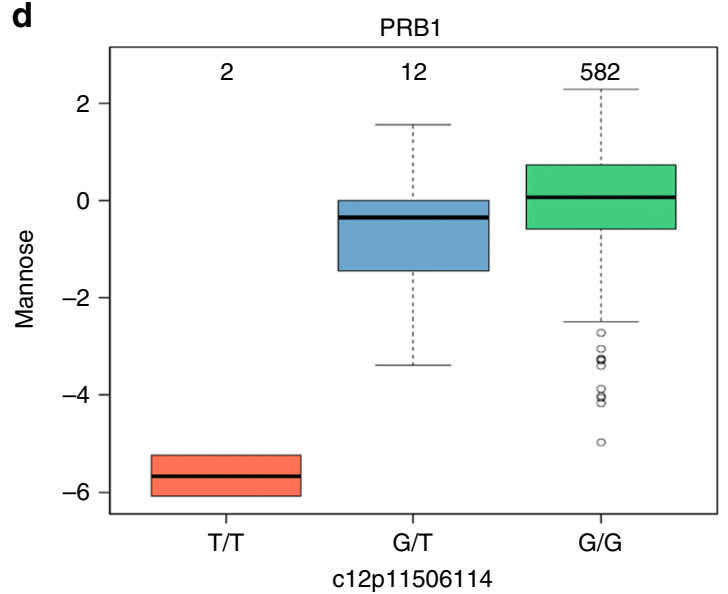

$\mathbf{f}$

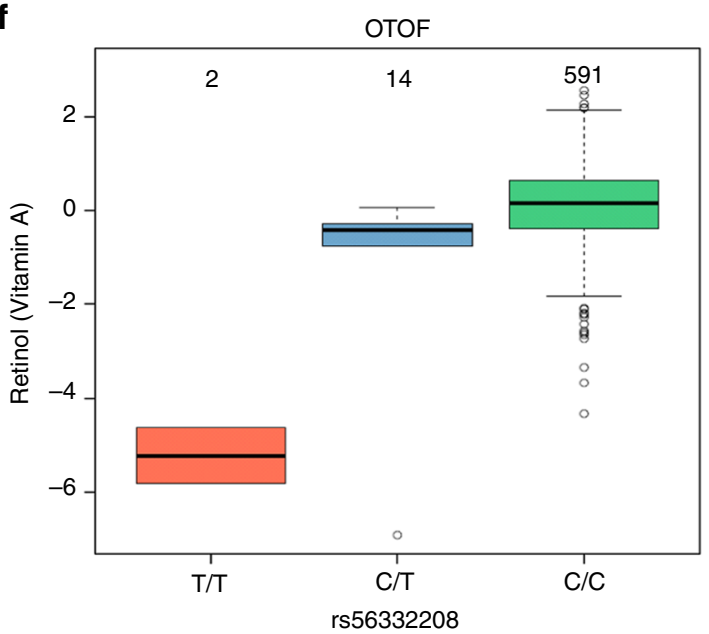

Fig. 6 Boxplots for the rare variant loci $A A S D H, P R B 1, A C A N$, and OTOF, indicating the metabolite level and the number of samples for each genotype group. Boxplots of rare variants associations of AASDH with thyroxine (3 SNPs) (a-c), PRB1 with mannose (d), ACAN with X-12844 (e), and OTOF with retinol (vitamin A) (f)

metabolism ${ }^{22}$; specifically, slow acetylation was reported for individuals harboring either of the two missense SNPs rs1799930 and rs1801280 (MIM: 612182.0001 and 612182.0002). Additionally, we discovered associations between SLC17A1 and the ratio hexanoylglutamine/X-16087. Previously, rs1185567 in SLC17A3 was reported to be associated with steroid levels ${ }^{11}$. However, since the SLC17 family are vesicular glutamate transporters $^{37}$, our association signal, linking the missense variant p.Thr269Ile (rs1165196) to a ratio containing hexanoylglutamine may reflect a direct biological relationship. We also discovered mQTLs associating known loci to metabolites in pathways different from those previously reported. Once such example is the association between the missense variant rs2147896 (p. Met461Thr) in PYROXD2 with levels of $N$-methylpipecolate. This 
gene had previously been linked to levels of urinary trimethylamine $^{18}$ and dimethylamine $e^{5}$, yet interestingly not found significantly associated with any of the mentioned metabolites in previous large scale studies ${ }^{11,15}$. Finally, our study expands the associations of several loci with newly identified metabolites or ratios as in the NAT2 locus and FADS2 locus among others (Supplementary Data 5). One example that supports the functional importance of the discovered loci is the SLCO1B1 locus. Its sentinel SNP, rs4149056, was previously associated with the ratio isoleucine/X-11529 11 , and other unknowns, and found in our study to be associated with the bile acids glycochenodeoxycholate-glucuronide(1) (which was also recently found to be the retired form of X-11529 by Metabolon), and glycocholenate sulfate. That supports the functionality of SLCO1B1 as a bile acid transporter, suggesting that the mutation p.Val174Ala alters the gene's native function ${ }^{38}$.

In addition to common mQTLs, we discovered a total of 12 rare mQTLs- 10 by single-variant analysis and 2 by gene-burden analysis-with interesting biological implications (Table 3 and Supplementary Fig. 5). First, the ACAN gene encodes a major component of the extracellular matrix, lending important biomechanical properties of cartilage, which explains its role in diseases such as osteoarthritis (MIM: 155760). We discovered an association of a missense variant p.Ala766Thr ( $\mathrm{rs} 150988100)$ in $A C A N$ with a steroid (X-12844, tentatively identified by Metabolon as glucuronidated steroid); it is well-established that circulating steroid levels are linked to inflammation in joints and diseases such as arthritis ${ }^{39-42}$. Additional investigations would be needed to uncover the relation between the variant and the metabolite since one of the two subjects having this mutation have musculoskeletal problems. Similarly, the association of rs377301648 in ZNF133 with a 3-methyl-2-oxovalerate, a metabolite in the branched-chain amino-acid pathway might be due to the role of the gene in osteoblast differentiation ${ }^{43}$ and the previously reported involvement of 3-methyl-2-oxovalerate in osteoarthritis ${ }^{44}$. Additionally, association of the c12p11506114 mutation in PRB1 with mannose (Fig. 6) might be due to the involvement of this glycosylated, proline-rich protein in the salivary secretion pathway ${ }^{45}$, (Michael W King, PhDlఠ 1996-2016 themedicalbiochemistrypage.org ${ }^{46}$.

Another interesting rare variant association is that between rs56332208 in OTOF with retinol (vitamin A). This might be a disease-relevant link because retinoic acid mediates the regeneration of specialized mechano-sensory hair cells in the inner ear that capture auditory and balance sensory inputs, and which die after acoustic trauma, ototoxic drugs or aging diseases, leading to progressive hearing loss ${ }^{47}$. Previous studies have also described mutations in OTOF causing recessive neurosensory nonsyndromic deafness in patient cohorts from many different ethnicities via the gene's role in exocytosis of inner and outer hair cells $^{48-52}$. Thus, our study may provide a mechanistic link between this gene and hair cell development via modulation of retinol levels. Finally, burden-testing revealed mQTLs in AASDH and METTL7B associated with decreased levels of thyroxine and androsterone sulfate respectively. The $A A S D H$ gene plays an active role in the pipecolate pathway in which thyroxine is a byproduct $^{53}$. Thus, these mutations may impact protein function leading to significant reduction of thyroid hormone and metabolism. Regarding the METTL7B mQTL, one of the three mutations in this gene (rs115687886) is a nonsense mutation, suggesting the other two are also loss-of-function mutations as they influence the metabolite in the same direction. Increases in METTL7B expression had been previously observed in patients with acute respiratory distress syndrome, involving tissue injury and inflammation ${ }^{54}$, whereas adrenal androgens as androsterone sulfate have been observed to decrease in stress and inflammation ${ }^{39-42}$. The fact that one of the two subjects with the rs199581976 mutation had recurrent chest infections and pneumonia and the other subject had bronchial asthma, recurrent pneumonia, and right lung lower lobe collapse, supports the functionality of the gene, and thus provides another possible link between a rare variant and disease via metabolite-level modulation.

To summarize, our study revealed 21 mQTLs in Qataris, among which 7 are unknown to studies that used older metabolomics platforms, 10 are metabolically fine-mapped with new metabolite ratios, and 11 in which the sentinel SNP was at or in complete LD with protein-altering variants. We also discovered 12 novel functional rare variant mQTLs that are likely specific to this ethnic population. We believe that it is important to replicate rare variant associations, yet the low frequency of these variants and absence of similar cohorts makes it challenging. This study demonstrates the efficiency of using WES for mQTL discovery, which could be more convenient compared to the more expensive WGS, while providing deep coverage of protein coding variants, for fine-mapping of common mQTLs and being suitable for rare variant $\mathrm{mQTL}$ discovery. The use of WES is strongly supported by the ability to replicate a fair fraction of the mQTLs in Caucasians (19\% of all loci and $35 \%$ of replicable loci). Finally, this study is the largest and the first of its kind in a Middle Eastern population, where we show that studies in consanguineous populations offer a large insight with modest sample sizes and have the potential to reveal loci linked to disease and pharmacological/drug targets important to precision medicine in this region of the world.

\section{Methods}

Study cohort. Human subjects were recruited and written informed consent was obtained at Hamad Medical Corporation (HMC) and HMC Primary Health Care Centers in Doha, Qatar and approved by the Institutional Review Boards of Hamad Medical Corporation and Weill Cornell Medicine in Qatar. Briefly, a total of 614 subjects were recruited for exome sequencing, and another 382 subjects for array genotyping. Subjects were included on the basis of being 3-generation Qataris (four grandparents born in Qatar) and being healthy or diabetics, above the age of 30 years. Sample demographic characteristics are given in Table 1.

Exome sequencing. DNA of 614 subjects was extracted from blood using the QIAamp DNA Blood Maxi Kit (Qiagen Sciences Inc, Germantown, MD) and subjected to exome sequencing on the Illumina HiSeq 2000 platform using standard methods. Each subject was sequenced to a minimum mean depth of 70X. Genotypes were generated using the GATK Best Practices workflow ${ }^{55}$ (Whole exome data for a set of recently received 64 samples were merged with those of the 550 samples after calling variants using GATK on each and finding that the majority of SNPs detected in both sets overlap. We limited the analysis to variants in both sets by setting a genotype call rate of $\geq 98 \%$ for common variant analysis and $90 \%$ for rare variant analysis). Detailed preparation methods and genotypes are available in ${ }^{56}$. The DNA of 382 subjects was extracted and subjected to array genotyping using Illumina Omni $2.5 \mathrm{M}$ array kits.

Imputation and filtering. Exome and array data were imputed after being filtered (MAF $\geq 0.05, p_{\mathrm{HWE}}>10^{-6}$, genotype call rate $\geq 98 \%$ ) based on phased 108 Qatari whole genomes as a reference panel, using shapeit ${ }^{57}$ and Impute2 software packages ${ }^{58,59}$. Throughout all the manuscript and supplementary data sets/information, two unique notations of "c"\#"p"\# and \#:\# for indicating a chromosome, position pair (for example c1p100 and 1:100) are used to distinguish original exome SNPs and imputed exome SNPs, respectively. A total of 22 million SNPs were imputed into 614 Qatari Exomes. For common-variant association analysis, $1,650,892$ SNPs were used, after removing SNPs with imputation quality $R^{2}<0.5$ $\mathrm{MAF}<0.05$, genotype call rate $<98 \%$, and $\mathrm{pHWE}<10^{-6}$. For rare variant analysis, the methods are described separately below. To define a Bonferroni threshold for finding significant associations, we used the pruned set of SNPs at LD 0.8 (using plink command indep-pairwise 5050.8 ) that resulted in a total of 272,061 SNPs. Replication was performed in array-genotyped data from a separate set of 382 samples, where imputation using the same 108 Qatari genomes produced a total of 11 million high-quality SNPs.

Metabolomics data. Serum samples were prepared for metabolomics analysis as follows: $200 \mu$ l of serum were aliquoted, barcoded and transported on dry ice to 
Metabolon Inc. for analysis. Some samples were found hemolyzed, and the degree of hemolysis was recorded for each sample (according to a hemolysis chart as given by Metabolon Inc.), for correcting for its effect on metabolomics measurements. The Metabolon DiscoveryHD4 platform was used (details are given in Supplementary Note 1). This platform utilized a Waters ACQUITY ultra-performance liquid chromatography (UPLC) and a Thermo Scientific Q-Exactive high resolution/accurate mass spectrometer interfaced with a heated electrospray ionization (HESI-II) source and Orbitrap mass analyzer operated at 35,000 mass resolution. A total of 1303 metabolites were measured on that platform. Outlier metabolite measurements ( 3 standard deviations larger than the mean) were replaced by a missing value to avoid biasing the results. Metabolites with more than $20 \%$ missing values were removed from the data. A total of 826 metabolites (including 249 unknown metabolites) survived as high quality and observed in $>80 \%$ of all individuals. Metabolite measurements were $\log$-scaled and $z$-score normalized. Since samples were collected over different periods of time, and there was no adopted fasting criterion, we addressed that issue by investigating whether there are batch effects in the PCA of metabolic data, and did not find any grouping for any of the 614 exome samples or the 382 array samples. Several unknown metabolites were investigated by the aid of Metabolon, and which revealed their identities (see below).

Identification of unknown metabolites by Metabolon. Identification of tentative structural features for unknown biochemicals incorporates a detailed analysis of mass spec data, i.e., gathering information such as the accurate monoisotopic mass, the elution time and fragmentation pattern of the primary ion, and correlation to other molecules. The accurate monoisotopic mass is used to identify a likely structural formula for the unknown biochemical, which is then used to search against chemical structure databases (e.g., ChemSpider, SciFinder). When a candidate structure fits the accurate monoisotopic mass and fragmentation data, an authentic standard is commercially purchased or synthesized (when possible). Conformation of a proposed structure is based on a match to three primary criteria, including co-elution with the unknown molecule of interest, and a high degree match to both the accurate monoisotopic mass and fragmentation pattern. When a standard is not available to confirm the identity of the unknown biochemical but sufficient data exist to support a high degree of confidence in its identity, the unknown biochemical may be retired for a named structure that is differentiated from other named metabolites confirmed with authentic standards by the addition of an asterisk after the biochemical name. When a high degree of confidence in the identity of the unknown molecule is not obtained, the molecule retains its unnamed status as designated by an 'X-' in the molecule name. Refer to Supplementary Note 2 for the particular identification information on each of the identified unknown metabolites, and Supplementary Fig. 6 for the comparison of $\mathrm{MS}^{2}$ fragmentation spectrum and Extracted Ion Chromatogram of the candidate and unknown metabolite that were structurally confirmed by Metabolon.

mGWAS analysis. All associations were performed on imputed exome or imputed array genotype data. $P$-values and effect sizes were calculated using functions from both GenABEL and ProbABEL packages in R (version 3.1.2) that were used for computing associations between SNPs and metabolite/ratio levels (after being preprocessed as described in the previous sections) while correcting for age, gender, BMI, T2D, hemolysis, population stratification (using two PCA components) and kinship (for family relatedness). The "polygenic" function in GenABEL was used for correcting metabolite levels for covariates and kinship matrix, and regression analysis using "mmscore" function was used to find association between the residuals obtained from this function and the SNPs using an additive inheritance model. Heritability estimates are based on "esth2" values resulting from this function. Kinship matrix was computed based on the genotype data using the "ibd" function in GenABEL. The mean inflation factor for all metabolites was 0.98 . Percent of variance is calculated as follows: $r^{2}=\chi^{2} /\left(N-2+\chi^{2}\right)$, where $\chi^{2}=(\beta / \text { s.e. })^{2}$, $\beta$ and s.e. are the $\beta$ and the standard error of the $\beta$, as obtained from the regression results, and $N$ is the number of samples involved in this association. Regional association plots were produced using an in-house tool similar to that of (http:// snipa.helmholtz-muenchen.de/snipa/), yet based on LD of subjects in the exome data used here.

Loci and sentinel SNPs. Association results were divided into $500 \mathrm{Kbp}$ blocks, and in each of these the sentinel SNP and sentinel metabolite are defined according to the SNP-metabolite association with the highest significance. Those define the mQTLs. In the case where a locus had two significant SNPs not in LD (i.e., LD < 0.5 ) and lying in two different genes, it was broken down into two loci (as in the case of THEM4 gene and SPTLC1P4/AL591893.1, where those two loci are at LD= $0.3)$. However, where there are two SNPs not in $L D(L D<0.5)$ but are in the same gene, we did not split the locus and we report LD values for those SNPs (in NAT2 and FADS2 genes) in Supplementary Data 5. All functional variants-missense and splice variants reported are in LD with the sentinel SNP of the locus except for the NAT2 locus where the two missense SNPs are not in LD with the sentinel SNP. information than the two corresponding metabolite concentrations alone". It is calculated as: $p$-gain $=\min (p-\operatorname{val}(m 1), p-\operatorname{val}(m 2)) / p-\operatorname{val}(m 1 / m 2)$, given two metabolites $m 1$ and $m 2$, and the $p$-values ( $p$-val) of the association of the metabolite/ ratio to the same SNP. The $p$-gain threshold according to ${ }^{19}$ assumes a level of significance of $\alpha / B$, where a type I error rate of $\alpha=0.05$ is used, and the critical value for the $p$-gain is $B /(2 \cdot \alpha)$, i.e. $10 \cdot B$. Thus for Bonferroni correction of $B$ tests, the uncorrected critical value of 10 can be multiplied by the number of tests $B$. Accordingly, we set the value of $10 \times 826 \times 18$ (10×number of metabolites $\times$ number of loci obtained from single metabolites) as an approximate threshold (we took the maximum of the number of tests of the first and the second methods where the first method (as mentioned earlier) considers the ratio of the sentinel metabolite against all the rest of 825 metabolites, tested with SNPs in $100 \mathrm{~KB}$ of the sentinel SNP of this locus in each of the loci discovered by single-metabolite analysis, and the second method considers the ratio of two metabolites associated with the same SNP but with opposite beta signs, in this case 13,281 associations were tested).

Fisher exact test for randomness of missing values. This was used to detect whether the missing metabolite values from certain genotype groups $(A A, A B$, and $\mathrm{BB}$ ) were missing by chance, when studying the effect of a missense on the metabolite level (after making sure the missingness was not due to removing outliers in the pre-processing of metabolite levels). The two inputs for the Fisher's exact test are: (a) the number of missing values from each genotype group, and (b) the total number of samples in each genotype group. fisher.test method in R was used for this calculation. The low $p$-value indicates a low probability having these missing values by chance.

Metabolite regression analysis and partial correlations. To find associations between selected metabolites and other phenotypes as age (as in the case of X22162 and X-24513), the metabolite level is regressed against age, gender, BMI, hemolysis, T2D, and population stratification (for inclusion of ethnicity) for all 996 individuals using $\operatorname{lm}$ function in R. Bonferroni $p$-value is used to report a significant association with a phenotype $(p \leq 0.05 / 826)$. Partial correlations between two metabolites are calculated using the GeneNet package in R, and significant partial correlations are those which pass a Bonferroni $p$-value of $(p \leq 0.05 /(826 \times$ $825 / 2)$ ).

Rare variants analysis. High-quality rare variants were selected from the original non-imputed exome data, with $\mathrm{MAF} \leq 5 \%, p_{\mathrm{HWE}}>10^{-6}$, and genotype call rate $\geq 90 \%$. Single-variant analysis was done using GenABEL package in R (as mentioned above for common variants), and burden test was done using seqMeta package in $\mathrm{R}$ (as in refs. ${ }^{60,61}$ ). For burden tests, we accounted for the number of genes $\left(n=9823\right.$ genes; Bonferroni $\left.\mathrm{p} \leq 6.16 \times 10^{-9}\right)$, and for single-variant analysis, we accounted for the number of SNPs where we tested single-variant associations for SNPs with at least two homozygotes for the rare variant $(n=2660$ SNPs in 2119 genes; Bonferroni $p \leq 2.27 \times 10^{-8}$ ). All SNPs in the reported 12 rare variant mQTLs have a call rate of $100 \%$ except for $\mathrm{c} 12 \mathrm{p} 11506114$ with a call rate of $97 \%$. A filtering step was done by visual inspection of genotype-metabolite boxplots of significant associations to consider only associations where metabolite values of the minor homozygotes are at the extreme tail of the metabolite distribution (i.e., they should be the lowest or highest metabolite values for that metabolite).

URLs of databases used for annotation. GTEx portal (version 2.1, Build \#201) [www.gtexportal.org], OMIM diseases database [www.omim.org], Orphanet disease database [http://www.orpha.net] CHEMBL targets database [www.ebi.ac.uk/ chembl], PharmaGKB [www.pharmgkb.org], SNIPA [http://snipa.helmholtzmuenchen.de/snipa/], GenAtlas (http://genatlas.medecine.univ-paris5.fr/fiche.php? $\mathrm{n}=6643$ ), and GnomAD: http://gnomad.broadinstitute.org/.

Data availability. All information on metabolites are in Supplementary Data 1, expanded association results for 21 common variant mQTLs are in Supplementary Data 5, and the detailed biological annotations/interpretations of associations are in Supplementary Data 6, list of all associations $\left(p<=1.4 \times 10^{-7}\right)$ are in Supplementary Data 7 . All plots are also available in Supplementary Information and detailed information on Metabolon's method for identification of unknowns is available in the Supplementary Note. Exome data used in this project were selected from a pool of samples of which more than 1000 samples are deposited in SRA accessions SRP060765, SRP061943, and SRP061463, accessible online at http:// www.ncbi.nlm.nih.gov/Traces/study/?acc=SRP060765\%2CSRP061943\% 2CSRP061463\&go=go). (SRA accession SRP061943).

Received: 9 May 2017 Accepted: 30 October 2017 Published online: 23 January 2018 


\section{References}

1. Yousri, N. A. et al. Long term conservation of human metabolic phenotypes and link to heritability. Metabolomics 10, 1005-1017 (2014).

2. Beger, R. D. et al. Metabolomics enables precision medicine: "A white paper, community perspective". Metabolomics 12, 149 (2016).

3. Gieger, C. et al. Genetics meets metabolomics: a genome-wide association study of metabolite profiles in human serum. PLoS Genet. 4, e1000282 (2008).

4. Illig, T. et al. A genome-wide perspective of genetic variation in human metabolism. Nat. Genet. 42, 137-141 (2010).

5. Nicholson, G. et al. A genome-wide metabolic QTL analysis in Europeans implicates two loci shaped by recent positive selection. PLoS Genet. 7, e1002270 (2011).

6. Suhre, K. et al. A genome-wide association study of metabolic traits in human urine. Nat. Genet. 43, 565-569 (2011).

7. Draisma, H. H. et al. Genome-wide association study identifies novel genetic variants contributing to variation in blood metabolite levels. Nat. Commun. 6, 7208 (2015).

8. Kastenmuller, G., Raffler, J., Gieger, C. \& Suhre, K. Genetics of human metabolism: an update. Hum. Mol. Genet. 24, R93-r101 (2015).

9. Yet, I. et al. Genetic influences on metabolite levels: a comparison across metabolomic platforms. PLoS ONE 11, e0153672 (2016).

10. Kettunen, J. et al. Genome-wide study for circulating metabolites identifies 62 loci and reveals novel systemic effects of LPA. Nat. Commun. 7, 11122 (2016).

11. Shin, S. Y. et al. An atlas of genetic influences on human blood metabolites. Nat. Genet. 46, 543-550 (2014).

12. Demirkan, A. et al. Insight in genome-wide association of metabolite quantitative traits by exome sequence analyses. PLoS Genet. 11, e1004835 (2015).

13. Guo, L. et al. Plasma metabolomic profiles enhance precision medicine for volunteers of normal health. Proc. Natl Acad. Sci. USA 112, E4901-E4910 (2015).

14. Yazdani, A., Yazdani, A., Liu, X. \& Boerwinkle, E. Identification of rare variants in metabolites of the carnitine pathway by whole genome sequencing analysis. Genet. Epidemiol. 40, 486-491 (2016).

15. Long, T. et al. Whole-genome sequencing identifies common-to-rare variants associated with human blood metabolites. Nat. Genet. 49, 568-578 (2017).

16. Popejoy, A. B. \& Fullerton, S. M. Genomics is failing on diversity. Nature 538, 161-164 (2016).

17. Raffler, J. et al. Genome-wide association study with targeted and non-targeted nmr metabolomics identifies 15 novel loci of urinary human metabolic individuality. PLoS Genet. 11, e1005487 (2015).

18. Rueedi, R. et al. Genome-wide association study of metabolic traits reveals novel gene-metabolite-disease links. PLoS Genet. 10, e1004132 (2014).

19. Petersen, A. K. et al. On the hypothesis-free testing of metabolite ratios in genome-wide and metabolome-wide association studies. BMC Bioinformatics. 13, 120 (2012).

20. Lek, M. et al. Analysis of protein-coding genetic variation in 60,706 humans Nature 536, 285-291 (2016).

21. McKusick, V. A. Mendelian Inheritance in Man and its online version, OMIM. Am. J. Hum. Genet. 80, 588-604 (2007).

22. Whirl-Carrillo, M. et al. Pharmacogenomics knowledge for personalized medicine. Clin. Pharmacol. Ther. 92, 414-417 (2012).

23. Fakhro, K. A. et al. Rare copy number variations in congenital heart disease patients identify unique genes in left-right patterning. Proc. Natl Acad. Sci. USA 108, 2915-2920 (2011).

24. Jian, H., Liu, B. \& Zhang, J. Hypoxia and hypoxia-inducible factor 1 repress SEMA4B expression to promote non-small cell lung cancer invasion. Tumour Biol. 35, 4949-4955 (2014).

25. Lang, J. C. \& Schuller, D. E. Differential expression of a novel serine protease homologue in squamous cell carcinoma of the head and neck. Br. J. Cancer 84, 237-243 (2001).

26. Mackenzie, P. I. et al. Identification of UDP glycosyltransferase $3 \mathrm{~A} 1$ as a UDP $\mathrm{N}$-acetylglucosaminyltransferase. J. Biol. Chem. 283, 36205-36210 (2008).

27. Petersen, A. K. et al. Epigenetics meets metabolomics: an epigenome-wide association study with blood serum metabolic traits. Hum. Mol. Genet. 23 534-545 (2014)

28. van den Akker, E. B. et al. Meta-analysis on blood transcriptomic studies identifies consistently coexpressed protein-protein interaction modules as robust markers of human aging. Aging Cell 13, 216-225 (2014).

29. Menni, C. et al. Metabolomic markers reveal novel pathways of ageing and early development in human populations. Int. J. Epidemiol. 42, 1111-1119 (2013).

30. Veldhuisen, B., Spruit, L., Dauwerse, H. G., Breuning, M. H. \& Peters, D. J. Genes homologous to the autosomal dominant polycystic kidney disease genes (PKD1 and PKD2). Eur. J. Hum. Genet. 7, 860-872 (1999).
31. Sekula, P. et al. A Metabolome-Wide Association Study of kidney function and disease in the general population. J. Am. Soc. Nephrol. 27, 1175-1188 (2016).

32. UniProt Consortium. UniProt: a hub for protein information. Nucleic Acids Res. 43, D204-D212 (2014).

33. Kanehisa, M. \& Goto, S. Kegg: kyoto encyclopedia of genes and genomes. Nucleic Acids Res. 28, 27-30 (2000).

34. Corydon, M. J. et al. Role of common gene variations in the molecular pathogenesis of short-chain acyl-CoA dehydrogenase deficiency. Pediatr. Res. 49, 18-23 (2001).

35. van Maldegem, B. T. et al. Clinical, biochemical, and genetic heterogeneity in short-chain acyl-coenzyme A dehydrogenase deficiency. JAMA 296, 943-952 (2006).

36. Hunter-Zinck, H. et al. Population genetic structure of the people of Qatar. Am J. Hum. Genet. 87, 17-25 (2010).

37. Omote, H., Miyaji, T., Hiasa, M., Juge, N. \& Moriyama, Y. Structure, function and drug interactions of neurotransmitter transporters in the postgenomic era. Annu. Rev. Pharmacol. Toxicol. 56, 385-402 (2016).

38. Xiang, X. et al. Effect of SLCO1B1 polymorphism on the plasma concentrations of bile acids and bile acid synthesis marker in humans. Pharmacogenet. Genomics 19, 447-457 (2009).

39. Cutolo, M. Androgens in rheumatoid arthritis: when are they effectors? Arthritis Res. Ther. 11, 126 (2009).

40. Cutolo, M. et al. New roles for estrogens in rheumatoid arthritis. Clin. Exp. Rheumatol. 21, 687-690 (2003).

41. Cutolo, M. et al. Androgens and estrogens modulate the immune and inflammatory responses in rheumatoid arthritis. Ann. N. Y. Acad. Sci. 966, 131-142 (2002)

42. Cutolo, M., Sulli, A., Pizzorni, C., Craviotto, C. \& Straub, R. H. Hypothalamicpituitary-adrenocortical and gonadal functions in rheumatoid arthritis. Ann. N. Y. Acad. Sci. 992, 107-117 (2003).

43. Stains, J. P. \& Civitelli, R. Genomic approaches to identifying transcriptional regulators of osteoblast differentiation. Genome Biol. 4, 222 (2003).

44. Adams, S. B. Jr. et al. Global metabolic profiling of human osteoarthritic synovium. Osteoarthritis Cartilage 20, 64-67 (2012).

45. Stubbs, M. et al. Encoding of human basic and glycosylated proline-rich proteins by the PRB gene complex and proteolytic processing of their precursor proteins. Arch. Oral Biol. 43, 753-770 (1998).

46. Mandel, I. D. in The Glycoconjugates (ed. Horowitz, M. I. \& Pigman, W.) 153-179 (Academic Press, 1977).

47. Rubbini, D., Robert-Moreno, A., Hoijman, E. \& Alsina, B. Retinoic acid signaling mediates hair cell regeneration by repressing p27kip and sox2 in supporting cells. J. Neurosci. 35, 15752-15766 (2015)

48. Stelzer, G. et al. The GeneCards suite: from gene data mining to disease genome sequence analyses. Curr. Protoc. Bioinform. 54, 1.30.1-1.30.33 (2016)

49. Yasunaga, S. et al. A mutation in OTOF, encoding otoferlin, a FER-1-like protein, causes DFNB9, a nonsyndromic form of deafness. Nat. Genet. 21, 363-369 (1999)

50. Mirghomizadeh, F. et al. Substitutions in the conserved C2C domain of otoferlin cause DFNB9, a form of nonsyndromic autosomal recessive deafness. Neurobiol. Dis. 10, 157-164 (2002).

51. Adato, A., Raskin, L., Petit, C. \& Bonne-Tamir, B. Deafness heterogeneity in Druze isolate from the Middle East: novel OTOF and PDS mutations, low prevalence of GJB2 35delG mutation and indication for a new DFNB locus. Eur. J. Hum. Genet. 8, 437-442 (2000)

52. Choi, B. Y. et al. Identities and frequencies of mutations of the otoferlin gene (OTOF) causing DFNB9 deafness in Pakistan. Clin. Genet. 75, 237-243 (2009).

53. Hallen, A., Jamie, J. F. \& Cooper, A. J. Lysine metabolism in mammalian brain an update on the importance of recent discoveries. Amino Acids 45, 1249-1272 (2013).

54. Kovach, M. A. et al. Microarray analysis identifies IL-1 receptor type 2 as a novel candidate biomarker in patients with acute respiratory distress syndrome. Respir. Res. 16, 29 (2015).

55. DePristo, M. A. et al. A framework for variation discovery and genotyping using next-generation DNA sequencing data. Nat. Genet. 43, 491-498 (2011)

56. Fakhro, K. A. et al. The Qatar genome: a population-specific tool for precision medicine in the Middle East. Hum. Genome Var. 3, 16016 (2016).

57. Delaneau, O., Marchini, J. \& Zagury, J. F. A linear complexity phasing method for thousands of genomes. Nat. Methods 9, 179-181 (2011)

58. Howie, B. N., Donnelly, P. \& Marchini, J. A flexible and accurate genotype imputation method for the next generation of genome-wide association studies. PLoS Genet. 5, e1000529 (2009).

59. Howie, B., Marchini, J. \& Stephens, M. Genotype imputation with thousands of genomes. G3 1, 457-470 (2011).

60. Rhee, E. P. \& Yang, Q. An exome array study of the plasma metabolome. Nat. Commun. 7, 12360 (2016). 
61. Li, B. \& Leal, S. M. Methods for detecting associations with rare variants for common diseases: application to analysis of sequence data. Am. J. Hum. Genet. 83, 311-321 (2008).

62. Yousri, N. A. et al. Large scale metabolic profiling identifies novel steroids linked to rheumatoid arthritis. Sci. Rep. 7, 9137 (2017).

\section{Acknowledgements}

This work was supported by Biomedical Research Program (BMRP) in Weill Cornell Medicine-Qatar, funded by Qatar Foundation. It was also supported by Qatar National Research Funds (QNRF) grants numbers: 09-740-3-192, 09-741-3-793 and in part by grant number 7-272-1-041. We acknowledge the efforts of Greg Schaaf (Metabolon) for synthesizing standards used in the identification of structurally unknown molecules described in this manuscript.

\section{Author contributions}

N.A.Y., K.A.F., K.S. and R.G.C. conceived the study. N.A.Y. and K.A.F. led the study, interpreted genetic associations and wrote the paper. N.A.Y. performed all statistical and computational analysis, imputation, compilation and annotation of results, and interpretation of metabolic associations. K.A.F., J.L.R.-F., A.R., J.G.M. and R.G.C. provided all genotype and phenotype information for the Qatari subjects. R.P.M. provided Metabolon support for interpretation of unknown metabolites. A.A.-S. and O.M.C. performed sample collection and handling. S.A.K., T.O. and H.Z. aliquoted samples for Metabolon, and performed DNA extraction and processing in the genomic core. J.A.M., Y.A.M. and E.K.A. were responsible for the Genomic core for Illumina genotyping and NGS. G.T. provided pipeline tools for imputation and provided phased reference genome. M.K. provided plotting tools and aided with plotting results.

\section{Additional information}

Supplementary Information accompanies this paper at https://doi.org/10.1038/s41467017-01972-9.

Competing interests: R.P.M. is an employee of Metabolon Inc. The remaining authors declare no competing financial interests.

Reprints and permission information is available online at http://npg.nature.com/ reprintsandpermissions/

Publisher's note: Springer Nature remains neutral with regard to jurisdictional claims in published maps and institutional affiliations.

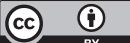

Open Access This article is licensed under a Creative Commons Attribution 4.0 International License, which permits use, sharing, adaptation, distribution and reproduction in any medium or format, as long as you give appropriate credit to the original author(s) and the source, provide a link to the Creative Commons license, and indicate if changes were made. The images or other third party material in this article are included in the article's Creative Commons license, unless indicated otherwise in a credit line to the material. If material is not included in the article's Creative Commons license and your intended use is not permitted by statutory regulation or exceeds the permitted use, you will need to obtain permission directly from the copyright holder. To view a copy of this license, visit http://creativecommons.org/ licenses/by/4.0/.

(C) The Author(s) 2017 